\title{
REVISIÓN TAXONÓMICA DEL GÉNERO CEPHALANTHERA (ORCHIDACEAE) EN LA PENÍNSULA IBÉRICA E ISLAS BALEARES
}

\author{
por \\ MARÍA LUISA ALARCÓN \& CARLOS AEDO \\ Real Jardín Botánico, CSIC. Plaza de Murillo, 2. E-28014 Madrid
}

\begin{abstract}
Resumen
AlARCón, M.L. \& C. AEDo (2002). Revisión del género Cephalanthera (Orchidaceae) en la Península Ibérica e Islas Baleares. Anales Jard. Bot. Madrid 59(2): 227-248.

Se revisa el género Cephalanthera en la Península Ibérica. Baleares y NW de África. Se aceptan tres especies: $C$. rubra, $C$. longifolia y $C$. damasonium. para las que se dan los sinónimos usados en la zona de estudio, descripción detallada y mapas de distribución. Se estudia el indumento, polen y semillas con el microscopio electrónico de barrido. Se neotipifica $C$. damasonium.

Palabras clave: Cephalanthera, Orchidaceae, taxonomía, distribución, tipificación, Península Ibérica, NW de África.
\end{abstract}

\begin{abstract}
AlARCón, M.L. \& C. AEDo (2002). A revision of the genus Cephalanthera (Orchidaceae) in the Iberian Peninsula and Balearic Islands. Anales Jard. Bot. Madrid 59(2): 227-248 (in Spanish).

A revision of the genus Cephalanthera in the Iberian Peninsula and NW of Africa has been done. Three species have been accepted. These species, $C$. rubra, C. longifolia and $C$. damasonium, have been listed with their synonyms in the study zone, a detailed description of each, and distribution maps. The indumentum pollen and seeds have been studied by SEM. $C$. damasonium has been typified.
\end{abstract}

Key words: Cephalanthera, Orchidaceae, taxonomy, distribution, typification, Iberian Peninsula, NW of Africa.

\section{INTRODUCCIÓN}

Se conocen 15 especies del género Cephalanthera distribuidas por las regiones templadas del Hemisferio Norte. En Norteamérica se encuentra una sola especie, $C$. austinae (Gray) Heller, que vive en los estados de la costa occidental de USA y es la única heterótrofa del género (LUER, 1975: 72). En Japón se han localizado tres especies: $C$. erecta (Thunb. ex Murray) Blume, C. falcata (Thunb. ex Murray) Blume y $C$. longibracteata Blume (ZOKU, 1965: 333). Las dos pri- meras se encuentran también en Corea y China (NAKAI, 1911: 221; ZHU GUANGHUA, 1999: 79) y la tercera alcanza además las regiones colindantes de Siberia (NEVSKII, 1935: 630). En China se conocen además $C$. bijianjensis S.C. Chen, C. calcarata S.C. Chen \& K.Y. Lang, y C. alpicola Fukuy., esta última al parecer endémica de Taiwan (ZHU GuANGHUA, 1999: 77-81). En torno a Persia, Cáucaso y Anatolia crecen otras cuatro especies: $C$. kurdica Bornm. ex Kraenzl., C. kotschyana Renz \& Taubenheim, C. caucasica Kraenzl. y C. epipactoides Fisch. \& C.A. Mey. (NEvSKII, 
1935: 633: DAVIS, 1984: 458; RENZ, 1978: 26), la última de las cuales se extiende también hasta Grecia (MoORE, 1980: 329). Otra especie del Mediterráneo oriental es $\mathrm{C}$. $\mathrm{Cu}$ cullata Boiss. \& Heldr., endémica de Creta (MOORE, 1980: 329). Finalmente, tres especies de más amplia distribución son las que se tratan en este artículo, $C$. damasonium, C. longifolia y $C$. rubra, que se distribuyen por el NW de África, la mayor parte de Europa y W de Asia. Estas especies han sido estudiadas en las floras locales y regionales de Europa (MOORE, 1980: 329) y N de África (MAI$\mathrm{RE}, 1959: 356$ ), pero no han sido objeto de una monografía reciente.

Este género necesita una revisión taxonómica especialmente en lo que respecta a las especies caucásicas y orientales, algunas de las cuales podrían quizá agruparse. La posición sistemática de Cephalanthera dentro de las Orchidaceae también necesita ser reevaluada. Según algunos autores, este género debe de ser adscrito a la tribu Neottieae, subfamilia Orchidoideae (DRESSLER, 1981). Un análisis filogenético basado en la secuenciación de un segmento cloroplástico sugiere que la tribu Neottieae es un grupo natural y que debe ser incluida en las Epidendroideae (CAMERON \& al., 1999). Otro análisis de esta naturaleza, basado en caracteres morfológi$\cos$, cuestiona la tribu Neottieae como grupo natural y sitúa Cephalanthera en una posición basal, sin resolver, tanto para las Orchidoideae como para las Epidendroideae (Freudenstein \& Rasmussen, 1999). Por otro lado, según PRUTSCH \& SCHILL (2001) la inclusión de Cephalanthera en la tribu Neottieae debe ser cuestionada, pues tienen un tipo de estigma típico de la subfamilia Epidendroideae.

En la Península Ibérica se han reconocido tradicionalmente las tres últimas especies señaladas, que son las extendidas por la mayor parte de Europa (WILlKomm 1861: 175). Se trata de especies bien delimitadas morfológicamente, aunque con una compleja sinonimia y con una distribución insuficientemente conocida en el área de estudio.

El objetivo de este trabajo es preparar una revisión taxonómica del género en la Penínsu- la Ibérica e Islas Baleares, en la que se integren la mayor parte de las recolecciones disponibles, para conocer con precisión la distribución geográfica y la variabilidad morfológica de estas especies. Se trata de una contribución con vistas a la preparación del volumen 21 de Flora iberica. Secundariamente, se han estudiado colecciones del Magreb, aunque con un menor esfuerzo en la búsqueda de material en los herbarios, con el objeto de aportar algo a la distribución de las Cephalanthera en una zona todavía escasamente prospectada como es el NW de África.

\section{MATERIAL Y MÉTODOS}

Para esta revisión se han estudiado más de 680 especímenes de los siguientes herbarios: ARAN, BC, BCF, BCN, COI, JACA, LEB, LISE, MA, MAF, MPU, SALA, SANT, SEV, VAL y herbario personal de Ó. Sánchez Pedraja. Además se han estudiado microfichas o imágenes procedentes de LINN y BM.

Se incluyen los sinónimos más frecuentes en la literatura que afecta a la zona de estudio. Cuando alguno de ellos se ha usado como nombre aceptado en las floras básicas consideradas en Flora iberica (cf. CASTROVIEJO \& al., 1986: XXI) se indica con un asterisco. Se señalan únicamente los tipos de los nombres aceptados.

Los datos de hábitat, altitud, fechas de floración, distribución y nombres vernáculos se refieren a la Península Ibérica y Baleares. Para la presentación de los mismos se siguen los criterios de Flora iberica (CASTROVIEJO \& al., 1986).

La longitud de la flor se mide desde la base del ovario hasta el ápice del sépalo dorsal. La longitud del ginostemo incluye la de las anteras. La bráctea de la flor basal es considerablemente más larga que el ovario y se mide en la antesis. Las demás van disminuyendo hacia el ápice de la inflorescencia. Por ello en las descripciones se han medido la bráctea basal y la tercera.

Los mapas de distribución se han preparado contando principalmente con el material estudiado. Se indican con otro símbolo las ci- 
tas bibliográficas de las provincias de las que no hemos tenido acceso a pliegos de herbario. A algunas localidades relativamente imprecisas se les ha asignado unas coordenadas geográficas aproximadas, para facilitar su ubicación a usuarios no familiarizados con la geografía ibérica.

Las muestras estudiadas con el microscopio electrónico de barrido (MEB), tres por especie, fueron tratadas con el método de punto crítico (COHEN, 1974), metalizadas con una capa de oro de 40-50 nm y examinadas con un JEOL-TSM T330A a $15 \mathrm{kV}$.

\section{CARACTERES MICROMORFOLÓGICOS}

Se han estudiado con el MEB el indumento de diversas zonas de la planta, el polen y las semillas con el objetivo de buscar potenciales caracteres micromorfológicos.

\section{Indumento de la inflorescencia,} sépalos y ovarios

Cephalanthera rubra es densamente pelosa en esas zonas, con pelos 100-300 $\mu \mathrm{m}$, simples, uniseriados, pluricelulares, glandulíferos, lisos, con un pie de 1-4 células y una célula apical poco engrosada (fig. la-c).

La inflorescencia de Cephalanthera longifolia es completamente glabra. Por el contrario, $C$. damasonium tiene inflorescencias con algunos pelos dispersos. Los sépalos y ovarios son casi glabros en ambas especies. Este escaso indumento esta formado en $C$. longifolia por pelos de 53-156 $\mu$ m (fig. Id-f) y en C. damasonium por pelos y de 93-102 $\mu \mathrm{m}$ (fig. lg-i), semejantes en todo a los de C. rubra, pero con un pie de 1-2 células.

\section{Polen}

El polen de las tres especies es muy seme-
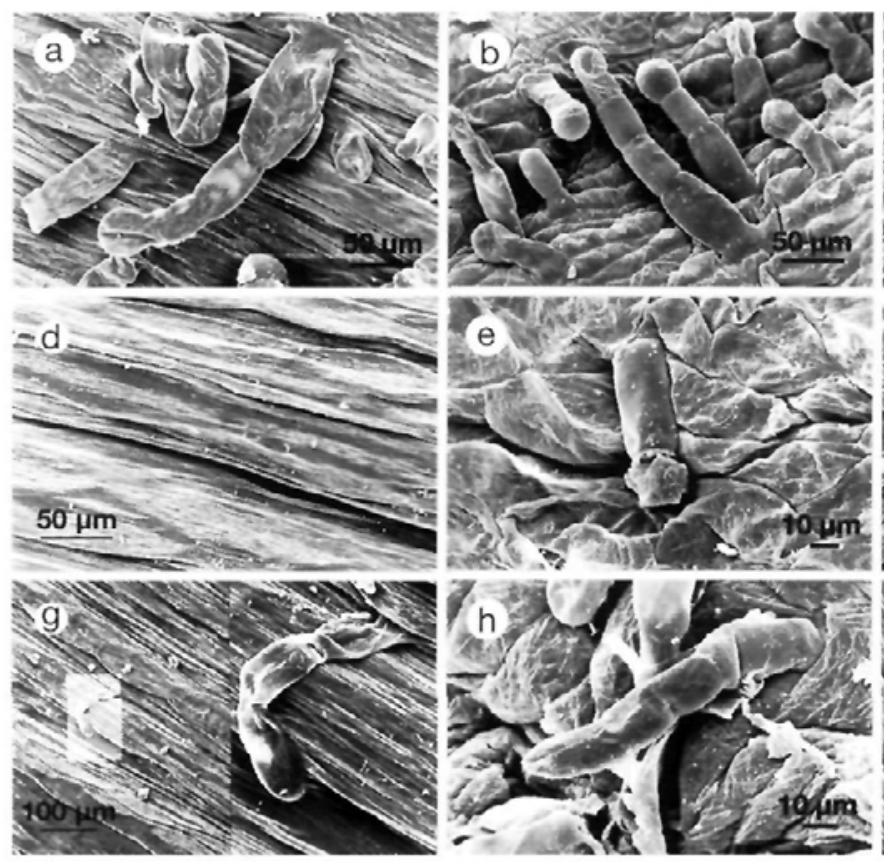

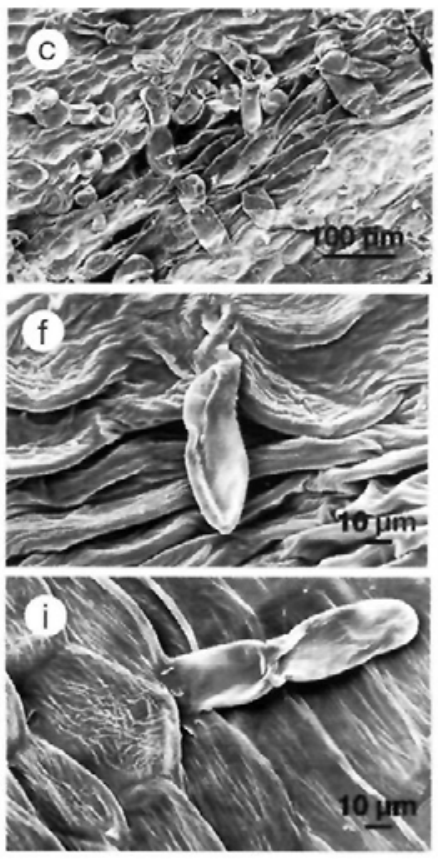

Fig. 1.-Microfotografías del indumento en Cephalanthera. C. rubra: a, inflorescencia (Jiménez \& al. 85, MA 626836); b, sépalo (Jiménez \& al. 85, MA 626836); c, ovario (Luceño s.n., MA 321753). C. longifolia: d, inflorescencia (Arizaleta \& al. 3042JP. MA 438751); e, sépalo (Soriano s.n., MA 462028); f, ovario (Lamata s.n., MA 587677). C. damasonium: g, inflorescencia (Gómez Manzaneque s.n., MA 453088); h, sépalo (Aedo s.n., MA 598359); i, ovario (Gómez Manzaneque s.n., MA 453088). 
jante, inaperturado, apolar, y con simetría radial. Nuestros resultados están de acuerdo con los de SuÁrez \& DíEZ (1987: 407) en C. rubra y $C$. longifolia. La superficie de la exina es reticulada con lúmenes de 1-2 $\mu \mathrm{m}$ y muros a veces tan anchos como los lúmenes (fig. 2).

\section{Semillas}

Las semillas son planas, reticuladas, con celdas alargadas. Tanto en la forma como en la ornamentación las tres especies son muy semejantes. Únicamente se ha podido apreciar alguna diferencia en la longitud de las semillas de $C$. damasonium, que llegan a tener $1,1 \mathrm{~mm}$, mientras que en $C$. rubra y $C$. longifolia no sobrepasan los $0,8 \mathrm{~mm}$ (fig. 3).

\section{BIOMETRÍA DE HOJAS Y BRÁCTEAS}

El tamaño relativo de las brácteas con respecto al ovario y la anchura de las hojas en relación con su longitud ha sido ampliamente usado para diferenciar estas especies, pero en general no ha sido cuantificado (MAIRE, 1959: 356; MOORe, 1980: 328; Clapham \& al., 1987: 559).

En la figura 4 se presentan los diagramas de cajas en los que se muestra la variación de los principales caracteres de hojas y brácteas. Las hojas de $C$. damasonium son más anchas en términos relativos, con una relación anchu$\mathrm{ra} /$ longitud $=0,24-0,5$, mientras que las de C. rubra y C. longifolia oscilan entre 0,05 y 0,36 . En la figura $4 a$ se puede ver que en la mayor parte de los casos la separación es clara.

Cephalanthera damasonium también se diferencia de las otras dos especies por su bráctea basal más ancha en relación a la longitud (relación anchura/longitud $=0,16-0,43$ ). Los valores de esta relación se solapan parcialmente en $C$. rubra y $C$. longifolia, aunque la figura $4 \mathrm{~b}$ muestra que $C$. longifolia tiene brácteas más estrechas en relación a la longitud.

La tercera bráctea de $C$. longifolia es mucho más corta que el ovario, con una relación longitud bráctea/ovario $=0,15-0,61$. Los valores de esta relación se solapan parcialmente en $C$. rubra y $C$. damasonium, aunque la figura $4 \mathrm{c}$ muestra que generalmente $C$. damasonium tiene brácteas más largas. En C. rubra la relación entre longitud de la tercera bráctea y la longitud del ovario varía entre 1 y 4 , en la mayor parte de los ejemplares estudiados. No obstante se han encontrado cuatro pliegos con brácteas mucho más largas, en los que esta relación va de 5 a 8,3. Dichos pliegos proceden de Castellón (MA 433916), Segovia (MA
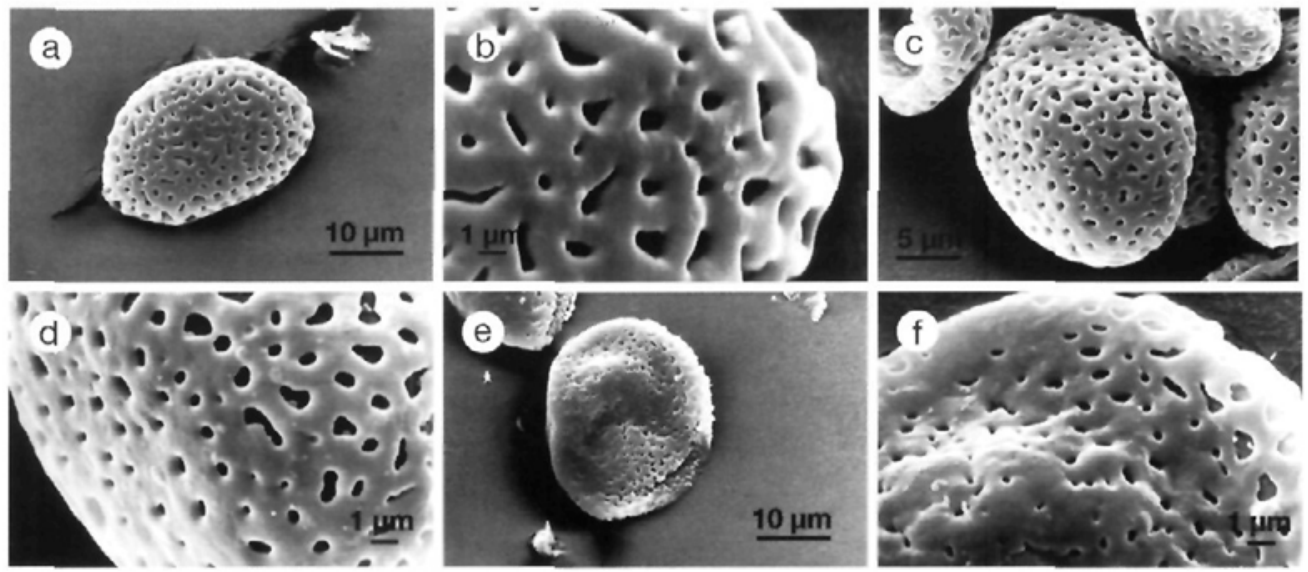

Fig. 2 -Microfotografías del polen de Cephalanthera: a,b, C. rubra (Luceño s.n., MA 321753); c,d, C. Iongifolia (Carrasco \& Pajarón s.n., MA 520669); e,f, C. damasonium (Carrasco \& al. 682FCL, MA 558807). 

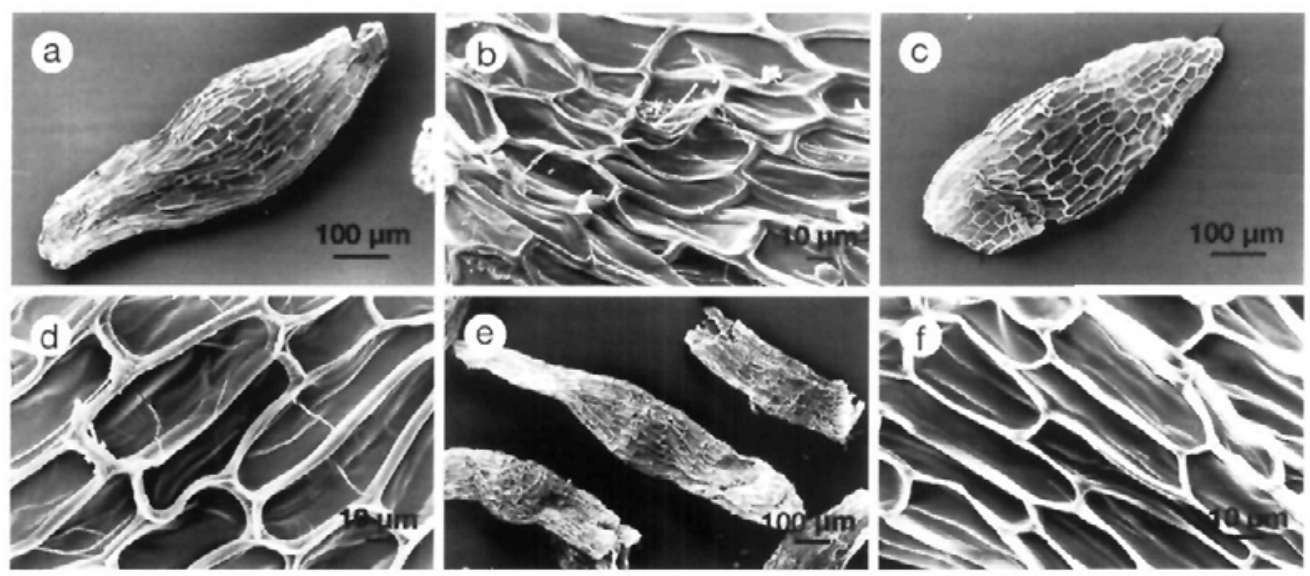

Fig. 3.-Microfotografías de las semillas de Cephalanthera. C. rubra: a (Pérez Carro s.n., LEB 14355); b (Caballero s.n., MA 24433); c,d, C. longifolia (López s.n., MA 393030); e,f, C. damasonium (Villar \& al. s.n., MA 515688).

560689), León (LEB 15044) y Guadalajara (MAF 36354). Parece que se trata de ejemplares excepcionales que por lo demás se adscriben sin dudas a $C$. rubra.

\section{TRATAMIENTO TAXONÓMICO}

\section{Cephalanthera Rich.}

Plantas perennes, herbáceas, generalmente autótrofas. Rizoma corto, con raíces \pm verticales fibrosas, fasciculadas. Tallos erectos, simples, cilíndricos, estriados, foliados, glabros o pelosos en el ápice. Hojas alternas, sentadas, envainadoras, de ovales a lanceoladas o lineares, con el margen entero, agudas, glabras, sin manchas. Inflorescencia en espiga terminal, erecta, laxa, con brácteas no envainantes, foliosas. Flores suberectas, sésiles o subsésiles, con las divisiones del perianto subiguales y conniventes. Sépalos erectos o erecto-conniventes, formando un casquete oblongo, agudo. Pétalos subiguales; labelo constreñido con una parte basal o hipoquilo, cóncavo, con lóbulos laterales erectos protegiendo al ginostemo, y una distal o epiquilo, oval y obtuso u oval-lanceolado y agudo, recurvado en el ápice, con 3-9 crestas longitudinales en la parte superior; sin espolón -en especies extraibéricas a veces con espolón corto-. Ginostemo alargado, \pm cilíndrico, casi tan largo como los pétalos, con rostelo indiferenciado; estigma en el ápice subredondeado, ancho, oblongo. Antera terminal libre, móvil, oblonga, con tecas biloculares; sin polinios, con masas polínicas bilobuladas, sésiles, desprovistas de retináculos; polen en mónadas. Ovario sésil o subsésil, cilíndrico, contorsionado. Fruto en cápsula erecta, oblonga. Semillas planas, reticuladas, con celdas alargadas.

1. Flores rosadas o púrpuras; eje de la inflorescencia y ovario con pelos glandulíferos; epiquilo agudo, oval-lanceolado, más largo que ancho .. 1. C. rubra

- Flores blancas o blanquecinas; eje de la inflorescencia y ovario glabros o casi glabros; epiquilo obtuso, más ancho que largo 2

2. Hojas 7-13 × 0,7-2(3) cm, linear-lanceoladas; brácteas medias y superiores mucho más cortas que el ovario; sépalos agudos ... 2. C. longifolia

- Hojas 4-9 × 1,4-3,8 cm, ovales; brácteas más largas que el ovario; sépalos obtusos ...

3. C. damasonium

1. Cephalanthera rubra (L.) Rich., De Orchid. Eur.: 38 (1817)

Serapias rubra L., Syst. Nat. ed.12, 2: 594 (1767) basión.; Epipactis rubra (L.) All., Fl. Pedem. 2: 153 (1785); Epipactis purpurea Crantz, Stirp. Austr. Fasc. 4: 457 (1769) 

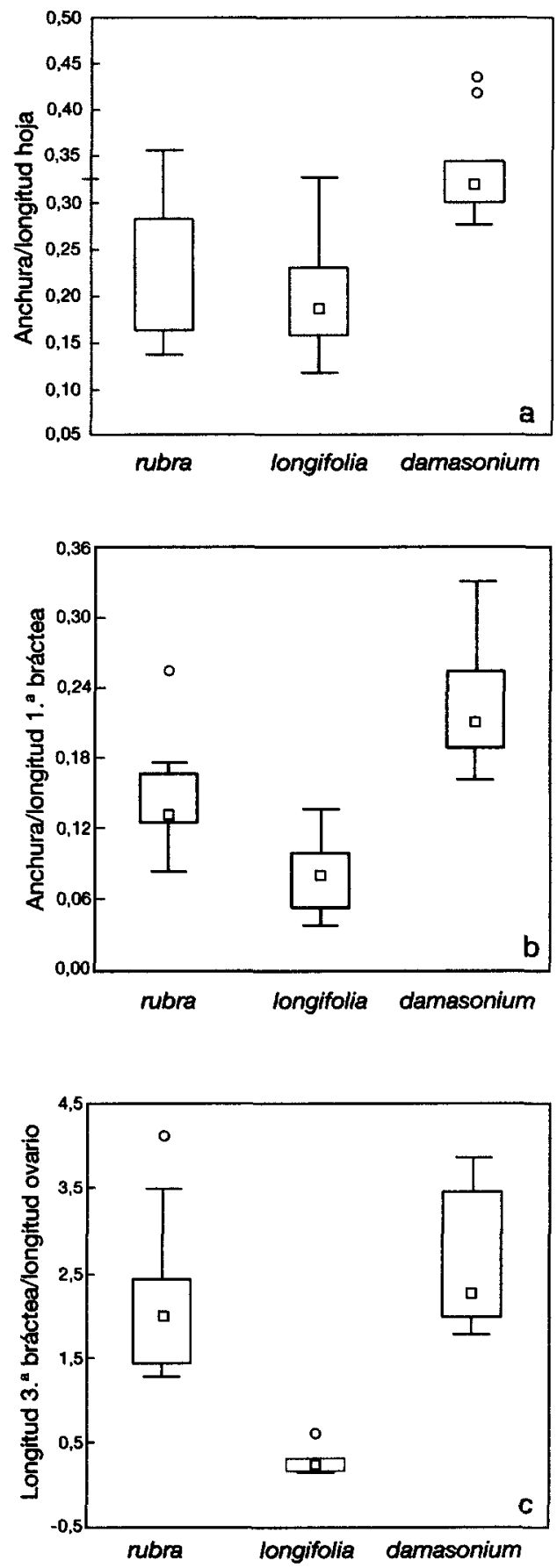

Fig. 4.-Diagramas de cajas con la variación de los principales caracteres de hojas y brácteas ( $n=10$ en todos los casos). [工 Non-OutlierMax/Not-Outlier Min; ㅁ 75\%/25\%; $\square$ Median; O Outliers].
Ind. loc.: [no indicada expresamente] [lectótipo designado por BAUMANN \& al., 1989: 574, LINN 1057.5, visto en microficha] Ic.: Ross-Craig, Draw. Brit. Pl. 28, pl. 43 (1971)

Rizoma corto, vertical, con abundantes raíces fibrosas, fasciculadas. Tallos $20-85 \mathrm{~cm}$, delgados, flexuosos, estriados, macizos, verdes, con pelos glandulíferos de $0,1-0,3 \mathrm{~mm}$ en la mitad superior. Hojas 4-5(7)-más 2-3 poco desarrolladas en la base-, de 5-10(13) $\times 1$ $2(3) \mathrm{cm}$, espaciadas, \pm lanceoladas -relación anchura/longitud $=0,14-0,36-$, agudas, glabras, más largas y estrechas cerca de la inflorescencia. Inflorescencia (3)6-15(24) $\mathrm{cm}, 3-$ 18 flores sésiles, con abundantes pelos glandulíferos de 100-300 $\mu \mathrm{m}$; bráctea de la flor basal 3-6 × 0,3-1 cm, lanceolada-relación anchura/longitud $=0,08-0.5-$, foliácea, agu$\mathrm{da}$, las restantes $1-3 \mathrm{~cm}$ de largo, igual o más largas que el ovario -relación longitud tercera bráctea/ longitud ovario $=1-2,25(4)-$, foliácea. lanceolada, aguda, con pelos glandulíferos dispersos por el envés - más abundantes en la base-, haz glabro. Flores $2-4 \mathrm{~cm}$ de longitud. Sépalos 1,2-2 × 0,4-0,7 cm, oval-lanceolados, agudos, con pelos glandulíferos dispersos en la cara externa, de un rosa \pm vivo. Pétalos laterales $(1,1) 1,2-2 \times(0,3) 0,4-0,7 \mathrm{~cm}$, oval-lanceolados, agudos, glabros, de un rosa \pm vivo; labelo $1,5-2 \mathrm{~cm}$, aproximadamente tan largo como los sépalos, sin espolón, glabro; hipoquilo 0,5-0,8 $\times 0,8-1 \mathrm{~cm}$, cóncavo, con dos lóbulos laterales triangulares agudos y dos lóbulos apicales que encierran el ginostemo, blanco con márgenes rosados; epiquilo 1$1,2 \times 0,6-0,7 \mathrm{~cm}$-más largo que ancho-, oval-lanceolado, agudo, de blanquecino a rosado, con (4)7-9 crestas longitudinales de color amarillo naranja. Ginostemo 0,6-1 cm, \pm cilíndrico, erecto, glabro. Antera c. $0,25 \mathrm{~mm}$, oval, algo cuculada, obtusa; masas polínicas blanquecinas. Ovario $1-1,5(2,3) \mathrm{cm}$ de longitud, con abundantes pelos glandulíferos. Fruto erecto, oblongo, con 6 costillas. Semillas $0,67-0,8 \times 0,2-0,3 \mathrm{~mm}$.

Números cromosomáticos: $2 n=36$ (VERLAQUE \& al., 1987; DRUSKOVIC \& LovKA, 
1995), $2 n=48$ (SEMERENKo, 1989; Ruiz, 1995).

Hábitat: Bosques caducifolios, encinares, pinares y abetales, generalmente en suelos calizos; (50)500-1600(1900) $\mathrm{m}$.

\section{Floración: (IV)V-VIII(IX).}

Área de distribución: Casi toda Europa, SW de Asia, Cáucaso, Anatolia, Persia y NW de África. Dispersa por casi toda la Península, salvo en algunas provincias occidentales, e Islas Baleares.

\section{Nombres vernáculos. cat.: curraià.}

Especie muy bien representada en el cuadrante NE de la Península, que se extiende hacia el W por las principales cadenas montañosas y alcanza Portugal en su extremo NW. En las Islas Baleares solo se conoce de Mallorca. En el $\mathrm{N}$ de África se encuentra en diversos puntos de Marruecos y es muy rara en Argelia (fig. 5).

No hemos podido estudiar material de las siguientes provincias: Asturias (cf. AEDO
\& al., 1990: 112), Badajoz (cf. PÉREz CHISCANO, 1977: 175), Granada (cf. MOLERo MESA \& al., 1981: 647; VALLE, 1981: 731) y Mallorca (cf. Ll. Sáez, in litt.).

\section{Material estudiado}

ALG, Djurdjura, Mont Tigormatin, $36^{\circ} 26^{\circ} \mathrm{N}, 4^{\circ} 13^{\prime} \mathrm{E}$, 8-VI-1930, R. Maire s.n., MA 24455.

SPA, ÁlAVA: Bernedo, $42^{\circ} 37^{\prime} \mathrm{N}, 2^{\circ} 30^{\prime} \mathrm{W}, \mathrm{VII}-1956$, M. Losa s.n., BCF 43876. ALBACETE: Padrón de Bienservida, $38^{\circ} 31^{\prime} \mathrm{N}, 2^{\circ} 36^{\prime} \mathrm{W}, 4$-VII-1923, J. Cuatrecasas s.n., BC 60093. Alicante: Alcoy, El Carrascal de Alcoy, $38^{\circ} 42^{\prime} \mathrm{N}, 0^{\circ} 28^{\prime} \mathrm{W}, 18$-VII-1957, A. Rigual s.n., MA 373111. Alcoy, Fuente Roja, $38^{\circ} 42^{\prime} \mathrm{N}, 0^{\circ} 28^{\prime} \mathrm{W}, \mathrm{VI}-$ 1980, Mansanet \& Mateo s.n. VAL 118164. Benifallím, Serra del Rentonar, $38^{\circ} 40^{\prime} \mathrm{N}, 0^{\circ} 24^{\prime} \mathrm{W}, 10$-VI-1989, L. Serra s.n., VAL 118171. ALMERía: Sierra María, $37^{\circ} 40^{\prime} \mathrm{N}, 2^{\circ} 13^{\prime} \mathrm{W}, 12-\mathrm{VII}-1960$, S. Rivas Goday s.n., MAF 83255. ÁvILA: El Tiemblo, 40⒉ $5^{\prime} \mathrm{N}, 4^{\circ} 30^{\circ} \mathrm{W}, 17-$ VI-1990, J.E. Echevarria \& R. Gavilán s.n., MA 562387. Hoyocasero, $40^{\circ} 24^{\prime}$ N, $4^{\circ} 58^{\prime}$ W, 21-VI-1983, M. Luceño s.n., MA 321753. Barcelona: Aguilar de Segarra, $41^{\circ} 45^{\prime} \mathrm{N}, 1^{\circ} 38^{\prime} \mathrm{E}$, 9-VI-1952, A. López \& A. Marcos s.n., BC 118805. Castelldefels, $41^{\circ} 17^{\prime} \mathrm{N}, 1^{\circ} 58^{\prime} \mathrm{E}, 25-\mathrm{V}-1930$, s.n. SANT 3038. Coll dels Brucs, $41^{\circ} 35^{\prime} \mathrm{N}, 1^{\circ} 47^{\prime} \mathrm{E}, 20-$ VI-1976, I. Alvaro \& al. s.n., BC 633895. El Bruc, Anoia, $41^{\circ} 35^{\prime}$ N, $1^{\circ} 47^{\prime}$ E, 23-VI-1982, J. Nuet Badia \& J.M. Panareda s.n., BC 641003 . Horsavinyà, $41^{\circ} 41^{\prime} \mathrm{N}, 2^{\circ} 39^{\prime} \mathrm{E}$,

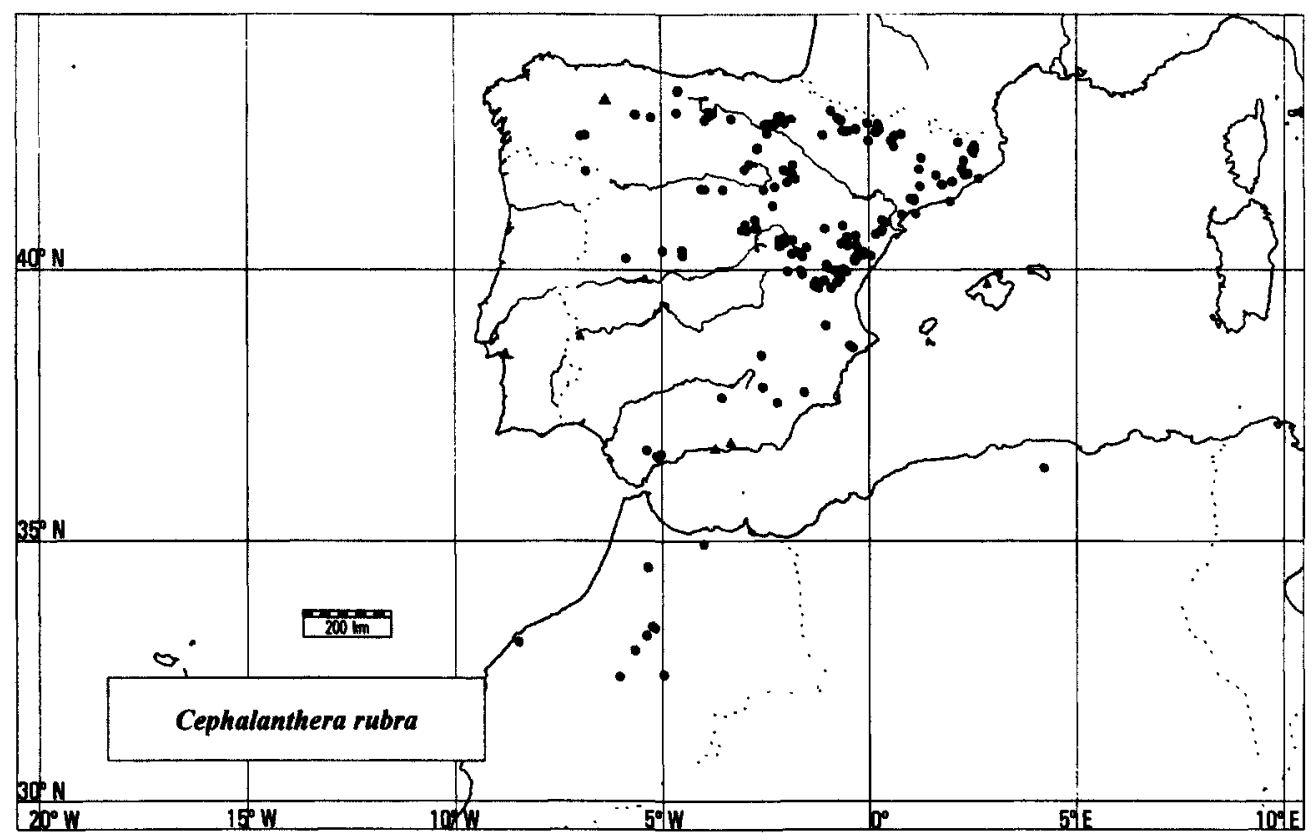

Fig. 5.-Distribución de Cephalanthera rubra en la Península Ibérica y N de África (๑, material estudiado; $\uparrow$, citas bibliograficas). 
30-VI-1946, P. Montserrat s.n., BC 620591. Macizo de Montserrat, $41^{\circ} 35^{\prime} \mathrm{N}, 1^{\circ} 46^{\prime} \mathrm{E}, 10$-VII-1946. P. Montserrat \& al. s.n., JACA 1446. A. Manlleu, $42^{\circ} 0^{\prime} \mathrm{N}, 2^{\circ} 17^{\prime} \mathrm{E}$ 20-VI-1926, Gonzalo s.n., BC 837527. Montseny, $41^{\circ} 46^{\circ} \mathrm{N}, 2^{\circ} 24^{\circ} \mathrm{E}$, J.M. Barnadés s.n., BC 603848. Santa Cecilia de Voltregà, $41^{\circ} 38^{\prime}$ N, $2^{\circ} 1^{\prime} \mathrm{E}, 27$-VII-1923, Gonzalo s.n., BC 837526. Tagamanent, $41^{\circ} 45^{\prime} \mathrm{N}, 2^{\circ} 19^{\prime} \mathrm{E}, 17$ VI-1947, Marcos s.n., BCF 3768. Tona, Osona, Turó del Castell, 41 ${ }^{\circ} 51^{\prime}$ N, $2^{\circ} 14^{\prime}$ 'E. 8-VI-1952, A. Bolòs \& O. Bolòs s.n., BC 118772. BuRGos: entre Escalada y Orbaneja del Castillo, $42^{\circ} 49^{\prime}$ N. $3^{\circ} 46^{\prime} \mathrm{W}, 5-\mathrm{VII}-1981$, J.A. Fernández Prieto s.n. MA 453193. Hoyos del Tozo, $42^{\circ} 42^{\prime} \mathrm{N}, 3^{\circ} 57^{\prime} \mathrm{W}, 15-\mathrm{VI}-1985$, J.A. Alejandre s.n., MA 339007. Monte en Barcina de los Montes y Penches. $42^{\circ} 43^{\prime}$ N, $3^{\circ} 19^{\prime}$ W, VI, $M$. Losa s.n., BCF 3769 . Orbaneja del Castillo. $42^{\circ} 50^{\prime}$ N, $3^{\circ} 47^{\prime}$ W, 8-VIII-1982. J. Pérez Carro s.n., LEB 14355. Sargentes de la Lora y S. Felices, $42^{\circ} 46^{\prime} \mathrm{N}, 3^{\circ} 52^{\prime} \mathrm{W}, P$. Galán Cela \& al. 1729PG, MA 204105. CÁCERES: Hervás, $40^{\circ} 16^{\prime} \mathrm{N}, 5^{\circ} 51$ 'W, 30-V1980, F.J. Fernández Diez \& al. s.n., SALA 25305. CÁDIZ: Grazalema, Sierra del Pinar, $36^{\circ} 46^{\prime}$ N. $5^{\circ} 22^{\prime} \mathrm{W}, 3-$ VI-1980, B. Cabezudo \& J. Díez s.n., SEV 88135. Grazalema, Sierra de Zafalgar, El Santo, $36^{\circ} 46^{\prime} \mathrm{N}, 5^{\circ} 22^{\prime} \mathrm{W}, 10$ VI-1993, A. Aparicio \& al. A.A.6704, MA 527019. CANTABRIA: Peña de la Ventosa, Lebeña, Cillorigo-Castro. $43^{\circ} 12^{\prime} \mathrm{N}, 4^{\circ} 36^{\prime} \mathrm{W}, 28-\mathrm{VI}-1984$, C. Aedo s.n., MA 598357. San Martín de Elines, bco. de Montecoza, pr. Villaescusa de Ebro, 42 ${ }^{\circ} 50^{\prime} \mathrm{N}, 3^{\circ} 51^{\prime} \mathrm{W}, 13-\mathrm{VII}-1994$, G. Moreno Moral \& al. s.n. (herb. Sánchez Pedraja-3593. Castellón: Alt Millars, Fuente la Reina, $40^{\circ} 4$ 'N, $0^{\circ} 37$ 'W, 3-VI-1989, J. Riera 949JRV, VAL 21538. Alt Palància, Pina de Montalgrao, $40^{\circ} 1^{\prime} \mathrm{N}, 0^{\circ} 39^{\prime} \mathrm{W}, 27$-VI1989, J. Riera I226JRV, VAL 21541. Baix de la Pegunta, Penyagolosa, $40^{\circ} 13^{\prime} \mathrm{N}, 0^{\circ} 21^{\prime} \mathrm{W}, 13-\mathrm{VI}-1958, \mathrm{M}$. Calduch s.n., VAL 118177. Bejís, Carrascal de Peñascabia. $39^{\circ} 54^{\prime} \mathrm{N}, 0^{\circ} 42^{\prime} \mathrm{W}, 10-\mathrm{VI}-2000$. J. Andrés \& al. 85JNT, VAL 42943. Benasal, barranco d'En Ramos, $40^{\circ} 23^{\prime} \mathrm{N}$, $0^{\circ} 8$ 'W, 4-VI-1994, C. Fabregat \& S. Lopez 6229CFSL, VAL 89859. Canales a La Salada, $39^{\circ} 50^{\prime} \mathrm{N}, 0^{\circ} 46^{\prime} \mathrm{W}, 21$ V-1982, A. Aguilella s.n., VAL 5994. Culla, La Mola, alrededores del Mas Branchat, $40^{\circ} 20^{\prime} \mathrm{N}, 0^{\circ} 10^{\prime} \mathrm{W}, 22$-VI1991, C. Fabregat \& S. Lopez 2216CF, VAL 89860. El Toro, barranco del Taconas, $39^{\circ} 59^{\prime} \mathrm{N}, 0^{\circ} 45^{\prime} \mathrm{W}, 24$-VI2000 , J. Andrés \& al. 90JNT, VAL 42962. Maestrat, Penyagolosa, $40^{\circ} 13^{\prime} \mathrm{N}, 0^{\circ} 21^{\prime} \mathrm{W}, 11-\mathrm{VII}-1957, A$. Bolos \& O. Bolòs s.n., BC 144784, montes de Fredes, $40^{\circ} 42^{\prime} \mathrm{N}$, $0^{\circ} 10^{\prime} \mathrm{E}, \mathrm{VI}-1979$, Mansanet \& Mateo s.n., VAL 71681. Peñagolosa, El Rodeznar, 40 $15^{\circ} \mathrm{N}, 0^{\circ} 21^{\prime} \mathrm{W}, 9-\mathrm{VI}-1999$, J. Jiménez \& al. 85, MA 626836. Puerto de Cabrillas, pr. Portell de Morella, $40^{\circ} 30^{\prime} \mathrm{N}, 0^{\circ} 18^{\prime} \mathrm{W}, 25$-VI-1988, G. Mateo s.n., VAL 59180. Sarratella, alrededores del Mas Atalaya, $40^{\circ} 19^{\prime}$ N, $0^{\circ} 2$ 'E, 15-VI-1991, C. Fabregat \& S. López 2104CF, VAL 89861. Villafranca del Cid, barranco de Horta, $40^{\circ} 25^{\prime} \mathrm{N}, 0^{\circ} 15^{\prime} \mathrm{W}, 20-\mathrm{VII}-1991, C$. Fabregat \& $S$. López $2292 C F$, VAL 89862 . Vistabella del Maestrazgo, $40^{\circ} 18^{\prime} \mathrm{N}, 0^{\circ} 17^{\prime} \mathrm{W}$, VII-1910, F. Beltrán s.n., MA 24434. CUENCA: Boniches, $39^{\circ} 59^{\prime} \mathrm{N}, 1^{\circ} 37^{\prime} \mathrm{W}, 28-$ VI-1956, C. Vicioso s.n., MA 165280. Cañete, rambla Maloja, $40^{\circ} 3^{\prime} \mathrm{N}, 1^{\circ} 39^{\prime} \mathrm{W}, 27-\mathrm{V}-2000$, VAL 42735 . Carrascosa, $40^{\circ} 36^{\prime} \mathrm{N}, 2^{\circ} 10^{\prime} \mathrm{W}, 16-\mathrm{VI}-1935$, Rivas s.n., MA 24432. Fuertescusa, $40^{\circ} 29^{\prime} \mathrm{N}, 2^{\circ} 10^{\prime} \mathrm{W}, 15-\mathrm{VI}-1982$, E. Rico \& J. Sánchez s.n., MA 311140 . Hoz de Beteta, $40^{\circ} 34^{\prime} \mathrm{N}, 2^{\circ} 4^{\prime} \mathrm{W}, 18-\mathrm{V}-1933$, A. Caballero s.n., MA 24433. Las Torcas, $40^{\circ} 2^{\prime}$ N, $1^{\circ} 58^{\prime} \mathrm{W}, 8-\mathrm{VI}-1974$, A. González \& al. 1382GF, MA 392985. Masegosa, $40^{\circ} 33^{\prime} \mathrm{N}$, $2^{\circ} 1$ 'W, 25-VI-1984, J.M. Herranz s.n., MA 330596. Pico Mazmorra, Aliaguilla, $39^{\circ} 47^{\circ} \mathrm{N}, 1^{\circ} 20^{\circ} \mathrm{W}, \mathrm{VI}-1978, G$. Mateo s.n., VAL 118176. Pico Ranera, Talayuelas, $39^{\circ} 51^{\prime}$ N, $1^{\circ} 17^{\prime}$ W, 2-VII-1976, G. Mateo s.n., VAL 118175. Solán de Cabras, $40^{\circ} 30^{\prime}$ N, $2^{\circ} 7^{\prime}$ W, 5-VI-1975, G. López \& al. 1215GF, MA 392975. Talayuelas, $39^{\circ} 51^{\prime} \mathrm{N}, 1^{\circ} 17^{\prime} \mathrm{W}, \mathrm{VII}-1976$, G. Mateo s.n., VAL 118170. Tragacete, $40^{\circ} 21^{\circ} \mathrm{N}, 1^{\circ} 51^{\circ} \mathrm{W}, 9-\mathrm{VI}-1974$, A. González \& al. 1393GF, MA 392988. Gerona: Gamotxa, Croscat de Sa-Cot. $42^{\circ} 8^{\prime}$ N. $2^{\circ} 32$ 'E. 26-VI-1952, A. Bolòs \& O. Bolòs s.n., BC 118254. Garrotxa, Oix, $42^{\circ} 16^{\prime} \mathrm{N}, 2^{\circ} 32^{\prime} \mathrm{E}, 13-\mathrm{VI}-1982$, J. Nuet Badia s.n., BC 643660. Olot, Sant Julià, $42^{\circ} 11^{\prime}$ N, $2^{\circ} 29^{\prime}$ E. A. Bolòs s.n., BC 145346. Serra de Prades, vall de Castellfollit, $42^{\circ} 13^{\prime} \mathrm{N}, 2^{\circ} 33^{\prime} \mathrm{E}, 20-\mathrm{VI}-1947$, o. Bolòs s.n., BC 104895. Vall de Ribes, vora Estèguel, $42^{\circ} 19^{\prime} \mathrm{N}, 2^{\circ} 9^{\prime} \mathrm{E}, 11$-VI1970, J. Vigo s.n., BC 605783. GuadalajaRA: Barriopedro, $40^{\circ} 48^{\prime} \mathrm{N}, 2^{\circ} 45^{\prime} \mathrm{W}, 6$-VI-1970, Bellot \& al. s.n., MA 95259. Barriopedro, $40^{\circ} 48^{\prime} \mathrm{N}, 2^{\circ} 45^{\prime} \mathrm{W}, 6-\mathrm{VI}-1970, \mathrm{Be}$ llot \& al. s.n., SALA 6187. A. Cañizar, $40^{\circ} 46^{\prime} \mathrm{N}, 3^{\circ} 4^{\prime} \mathrm{W}$, 18-VI-1969, Bellot \& Ron s.n., MA 95258. Chequilla, base del Cerro de Las Carrasquillas, $40^{\circ} 36^{\prime} \mathrm{N}, 1^{\circ} 50^{\prime} \mathrm{W}$, 21-VI-1995, M.A. Carrasco \& al. 672 FCL, MA 558797. Fuentes de la Alcarria, $40^{\circ} 45^{\prime} \mathrm{N}, 2^{\circ} 57^{\prime} \mathrm{W}, 18$-VI-1969, Bellot \& Ron s.n., MA 95267. Mandayona. $40^{\circ} 57^{\prime} \mathrm{N}$, $2^{\circ} 45^{\prime}$ W, 4-VI-1970, Bellot \& al. s.n., MA 95264. Padilla de Hita, 40'52'N, $2^{\circ} 59^{\prime}$ 'W, 27-VI-1970, Bellot \& al. s.n., MA 95265. Poveda de la Sierra, $40^{\circ} 38^{\prime} \mathrm{N}, 2^{\circ} 2^{\prime} \mathrm{W}, 20$-VI1995, M.A. Carrasco \& al. 557FCL, MA 558869. Valderrebollo, $40^{\circ} 49^{\prime} \mathrm{N}, 2^{\circ} 43^{\prime} \mathrm{W}, 18-\mathrm{VI}-1969$, Bellot \& Ron s.n., MA 95266. TERUEL: Guadalaviar, Montes Universales, Sierra de Albarracín, $40^{\circ} 23^{\prime} \mathrm{N}, 1^{\circ} 43^{\circ} \mathrm{W}$, C. Pau s.n., MA 434032. HuesCa: Aragüés del Puerto, 42 $42^{\circ} \mathrm{N}$, $0^{\circ} 40^{\prime} \mathrm{W}, 9$-VIII-1965, P. Montserrat s.n., JACA 102165.

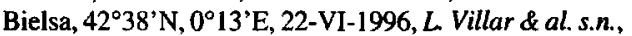
JACA 85996. Cajigar, $42^{\circ} 15^{\prime} \mathrm{N}, 0^{\circ} 36$ 'E, 26-VI-1992, J.V. Ferrández \& J.A. Sese s.n., JACA 126692. Hecho, Agüerri, $42^{\circ} 44^{\prime} \mathrm{N}, 0^{\circ} 45^{\circ} \mathrm{W}, 25-\mathrm{VII}-1974$, L. Villar s.n., JACA 10067674 A. Javierre del Obispo, 42 $33^{\prime} \mathrm{N}$, $0^{\circ} 19 '$ 'W, 29-VII-1988, D. Gómez \& L. Villar s.n., JACA 233388. Las Bellostas, $42^{\circ} 21^{\prime} \mathrm{N}, 0^{\circ} 1^{\prime} \mathrm{W}, 28-\mathrm{VI}-1983$, P. Montserrat \& J.M. Montserrat s.n., JACA 184683. Laspuña, $42^{\circ} 30^{\prime} \mathrm{N}, 0^{\circ} 10^{\prime} \mathrm{E}, 13-\mathrm{VII}-1956, P$. Montserrat \& N.Y. Sandwith s.n., JACA 9656 A. Oroel, Jaca, $42^{\circ} 3 I^{\prime} \mathrm{N}, 0^{\circ} 31^{\prime} \mathrm{W}, 1-\mathrm{IX}-1970$, P. Montserrat s.n., JACA 655670. San Juan de la Peña, $42^{\circ} 31^{\prime} \mathrm{N}, 0^{\circ} 38^{\prime} \mathrm{W}, 11$-VII1970, P. Montserrat \& L. Villar s.n., JACA 317170 . Saravillo, $42^{\circ} 33^{\prime} \mathrm{N}, 0^{\circ} 15^{\prime} \mathrm{E}, 21-\mathrm{VI}-1985$, G. Montserrat s.n., JACA 819985. Torla, Ordesa. Cotatuerto, $42^{\circ} 39^{\prime} \mathrm{N}$, $0^{\circ} 2$ 'W, 5-VIII-1971, P. Montserrat s.n., JACA 540571. Torre la Ribera, 42 $21^{\prime} \mathrm{N}, 0^{\circ} 32^{\prime} \mathrm{E}$, 11-VIII-1990, G. Montserrat \& J.A. Sesé s.n., JACA 415590. JAÉN: Almadén in mont. dict. Cabezaprieta, $38^{\circ} 46^{\circ} \mathrm{N}, 4^{\circ} 50^{\prime} \mathrm{W}$, 11-VI-1926, J. Cuatrecasas s.n., BC 60090. LA RIOJA: Almuerza, 21-VII-1984, C.L.M. s.n., SEV 161644. Logroño, $42^{\circ} 28^{\prime} \mathrm{N}, 2^{\circ} 27^{\prime} \mathrm{W}, 1799$, Pozo s.n., MA 24445. Nieva de Cameros, $42^{\circ} 13^{\prime} \mathrm{N}, 2^{\circ} 40^{\prime} \mathrm{W}, 6$-VII- 1982 , F. Amich s.n., SALA 26585. Rasillo de Cameros. $42^{\circ} 12^{\prime} \mathrm{N}, 2^{\circ} 42^{\prime} \mathrm{W}, \mathrm{VI}-1924$, MA 24446 B. LEÓN: La Ro- 
bla, $42^{\circ} 48^{\prime}$ N, $5^{\circ} 37^{\prime} \mathrm{W}, 26-\mathrm{VI}-1983, C$. Pérez Morales s.n., LEB 24935. Llanos de Alba, $42^{\circ} 48^{\prime} \mathrm{N}, 5^{\circ} 38^{\prime} \mathrm{W}, 19-$ VII-1988, E. Puente \& C. Pérez Morales s.n., LEB 38782. Peñas del Bufo, La Robla, $42^{\circ} 48^{\prime}$ N, $5^{\circ} 38^{\prime} \mathrm{W}, 6-$ VI-1985, M. Garzón s.n., LEB 15044. Valdemodiezmo, $42^{\circ} 45^{\prime}$ N, $5^{\circ} 15^{\prime} \mathrm{W}, 22-\mathrm{VI}-1988$, A. Penas \& al. s.n., LEB 38719. LÉRIDA: Peramola, ermita del Castellebre, $42^{\circ} 3^{\prime}$ N. $1^{\circ} 16^{\prime} \mathrm{E}, 11-\mathrm{VI}-1976, \mathrm{~J}$. Molero \& S. Silvestre s.n., MA 311956 . Vilamajor, $41^{\circ} 51^{\prime} \mathrm{N}, 1^{\circ} 13^{\circ} \mathrm{E}, \mathrm{VI}-1917$, M. Gallardo s.n., BC 125298. Alta Ribagorça, Vall de Boí, vora la Palanca de Saharís, $42^{\circ} 28^{\prime}$ N, $0^{\circ} 46$ 'E, 8-VII1978, E. Carrillo \& J.M. Ninot s.n., BCN 89. Vall Ferrera, Alins, vora Buiro, $42^{\circ} 26^{\circ} \mathrm{N}, 0^{\circ} 37^{\prime} \mathrm{E}, 23-\mathrm{VI}-1996, J . M$. Ninot s.n., BCN 92. MADRID: Las Rozas de Puerto Real, $40^{\circ} 19^{\prime} \mathrm{N}, 4^{\circ} 29^{\prime}$ W, 5-VII-1984, P. Montserrat \& J.L. González s.n., JACA 75284. MÁLAGA: Entre Ronda y San Pedro de Alcántara, cruce a Igualeja, $36^{\circ} 39^{\prime} \mathrm{N}$, $5^{\circ} 7$ 'W, 4-V-1989, Z. Díaz \& al. s.n., SEV 131267. Sierra de las Nieves, Pinsapar de Ronda, $36^{\circ} 42^{\prime} \mathrm{N}, 5^{\circ} 1^{\prime} \mathrm{W}, 9-$ VII-1930, C. Vicioso s.n., MA 24437. MURCIA: Sierra de Espuña. $37^{\circ} 52^{\prime} \mathrm{N}, 1^{\circ} 34^{\prime} \mathrm{W}, 12-\mathrm{VI}-1974$, E. Domínguez \& $S$. Talavera 2600/74. SEV 58889. NAVARRA: Ayegi. Montejurra, $42^{\circ} 39^{\prime}$ N, $2^{\circ} 2^{\prime}$ 'W, 4-VII-1980, Puy Isaba s.n., ARAN 48636. Azuelo, Kodes, barranco del Francés, $42^{\circ} 36^{\prime} \mathrm{N}, 2^{\circ} 21^{\prime} \mathrm{W}, 23-\mathrm{VI}-1985$, I. Aizpuru \& al. s.n., ARAN 32518. Barindano, Sierra Lokiz, $42^{\circ} 46^{\prime} \mathrm{N}$, $2^{\circ} 7$ 'W, 27-VI-1988, P. Garín s.n., ARAN 32520. Guirguillano, Orendain, $42^{\circ} 43^{\prime} \mathrm{N}, 1^{\circ} 52^{\prime} \mathrm{W}, 17-\mathrm{VI}-1985$, P. Garín s.n., ARAN 32522. Isaba, Artaparreta, $42^{\circ} 52^{\prime} \mathrm{N}$, $0^{\circ} 55^{\prime} \mathrm{W}, 24-\mathrm{VII}-1986$, I. Aizpuru \& Catalán s.n., ARAN 32519. Lapoblación, $42^{\circ} 36^{\circ} \mathrm{N}, 2^{\circ} 27^{\prime} \mathrm{W}, 18$-VIII- 1973. P. Montserrat \& al. s.n., JACA 485973. Marañón, $42^{\circ} 38^{\prime} \mathrm{N}, 2^{\circ} 26^{\prime} \mathrm{W}, 29$-VII-1980, Puy Isaba s.n., ARAN 48647. Nazar, Peña Gallet, $42^{\circ} 38^{\prime} \mathrm{N}, 2^{\circ} 16^{\prime} \mathrm{W}, 12$-VII1980, Puy Isaba s.n., ARAN 48645. Petilla de Aragón. $42^{\circ} 27^{\prime} \mathrm{N}, 1^{\circ} 7^{\prime} \mathrm{W} .24-\mathrm{VII}-1988$, I. Aizpuru \& Catalán s.n., ARAN 32521. Sierra de Santiago Loquiz, $42^{\circ} 45^{\prime} \mathrm{N}$, $2^{\circ} 11^{\prime}$ W, 30-VII-1987, Luceño \& Vargas PV2459, MA 351353. Torralba del Río, $42^{\circ} 36^{\prime} \mathrm{N}, 2^{\circ} 20^{\prime} \mathrm{W}, 18$-VI1973, L. Villar s.n., JACA 211173. ORENSE: Peñarrubia, $42^{\circ} 27^{\prime} \mathrm{N}, 6^{\circ} 50^{\prime}$ 'W. 2-VI-1981, F. Llamas s.n. LEB 13354. Rubiá, $42^{\circ} 26^{\prime} \mathrm{N}, 6^{\circ} 57^{\prime} \mathrm{W}, 22$-VI-1986, J. Amigo \& J. Giménez s.n. SANT 16363. Palencia: Peña Redonda, 42'50'N. 4'38'W, 20-VII-1988, M.E. García s.n., LEB 42816. SEGovia: Laguna de Contreras, $41^{\circ} 30^{\prime} \mathrm{N}$, $4^{\circ} 2^{\prime}$ W, 4-VII-1983, T. Romero s.n., MA 567040. Maderuelo, 41 $29^{\circ} \mathrm{N}, 3^{\circ} 31^{\prime} \mathrm{W}, 20-\mathrm{V}-1995$, Gabriel y Galán \& Puelles s.n., MA 560689 . Sacramenia, $41^{\circ} 30^{\prime} \mathrm{N}, 3^{\circ} 57^{\prime} \mathrm{W}$, 24-VI-1983, T. Romero s.n. SALA 40098. SORIA: Almazán, 41 ${ }^{\circ} 29^{\prime} \mathrm{N}, 2^{\circ} 32^{\prime} \mathrm{W}, 13-\mathrm{VI}-1933$, L Ceballos s.n., MA 24443. Bliecos, $41^{\circ} 32^{\prime} \mathrm{N}, 2^{\circ} 16^{\prime} \mathrm{W}, 24-\mathrm{VI}-1936$, C. Vicioso s.n., MA 24440 . Ciria, $41^{\circ} 37^{\prime} \mathrm{N}, 1^{\circ} 58^{\prime} \mathrm{W}, 20$ VI-1963, A. Segura Zubizarreta s.n., MA 359584. Covaleda, $41^{\circ} 56^{\prime}$ N, $2^{\circ} 53^{\prime} \mathrm{W}, 11$-VII-1935, L. Ceballos \& C. Vicioso s.n., MA 24442. Fuente la Teja, 30-VI-1975, A. Segura Zubizarreta s.n., MA 359587. Fuentes de Ágreda, Sierra de Moncayo, $41^{\circ} 48^{\prime} \mathrm{N}, 1^{\circ} 55^{\prime} \mathrm{W}, 20$-VII1936, C. Vicioso s.n., MA 24441. Matalebreras, $41^{\circ} 50^{\prime} \mathrm{N}$, $2^{\circ} 3^{\prime}$ W, 16-VI-1964, P. Moniserrat \& N.Y. Sandwith s.n.,

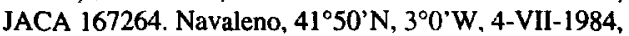
E. Rico \& T. Romero s.n., MA 567276. Somaen, $41^{\circ} 12^{\prime} \mathrm{N}, 2^{\circ} 19^{\prime} \mathrm{W}, 28-\mathrm{V}-1960$, A. Segura Zubizarreta
S.n., MA 359586. TARRagona: Horta de San Juan, Cami de las Heras, $40^{\circ} 57^{\prime}$ N. $0^{\circ} 19^{\prime} \mathrm{E}, 11-\mathrm{VI}-1982, \mathrm{~J}$. Molero \& A. Rovira s.n., BCF 70761 . Montalt, Llaberia, $41^{\circ} 3^{\prime} \mathrm{N}$, $0^{\circ} 48^{\prime} \mathrm{E}, 18-\mathrm{VI}-1973, \boldsymbol{R}$. Folch s.n., BC 627418. Muntanyes de Prades, hacia L'Espluga de Francolí, $41^{\circ} 20$ 'N, $1^{\circ} 6$ 'E, 8-VI-1950, E. Batalla s.n., BC 601384. Paüls, Serra d'Alfara, Baix Ebre, $40^{\circ} 55^{\prime} \mathrm{N}, 0^{\circ} 24^{\prime} \mathrm{E}$, 26-IV-1982, J. Molero \& A. Rovira s.n., BCF 70786. Pista de Fredes al monte Caro, base de la Mola del Boix, cara N, $40^{\circ} 47^{\prime} \mathrm{N}$, $0^{\circ} 19^{\prime} \mathrm{E}, 8-\mathrm{VI}-1999$, C. Navarro \& al. CN2404, MA 626807. Puertos de Beceite, El Retaule, $40^{\circ} 45^{\prime} \mathrm{N}$, $0^{\circ} 18^{\prime}$ E, 8-VI-1986, M.B. Crespo \& al. s.n. VAL 118168 A. Salou, $41^{\circ} 4^{\prime} \mathrm{N}, 1^{\circ} 8^{\prime} \mathrm{E}, F$. Sennen s.m., BC 837524 A. Tossal de la Baltasana, Prades, $41^{\circ} 20^{\prime} \mathrm{N}, 1^{\circ} 0^{\prime} \mathrm{E}, 13-\mathrm{VI}-$ 1982, A. Aguilella \& al. s.n., VAL 671. Vallfogona de Riucorb, $41^{\circ} 33^{\prime} \mathrm{N}, 1^{\circ} 14^{\prime} \mathrm{E}, 28$-VI-1918, M. Gallardo s.n., BC 125294. TerUeL: Alpeñés, $40^{\circ} 48^{\prime} \mathrm{N}, 1^{\circ} 4^{\prime} \mathrm{W}, 22-$ VI-1988, G. Mateo 1447, VAL 60071. Bordón, Masía de los Alcaldes. $40^{\circ} 40^{\prime} \mathrm{N}, 0^{\circ} 19^{\prime} \mathrm{W}, \mathrm{VI}-1981$, A. Aguilella s.n., VAL 10405. Camarena de la Sierra, Fuente el Cabrito, $40^{\circ} 9^{\prime} \mathrm{N}, 1^{\circ} 2^{\prime} \mathrm{W}, 1-\mathrm{VII}-1998, G$. Moreno Valdeolivas s.n., VAL 107198. Estercuel, $40^{\circ} 51^{\prime} \mathrm{N}, 0^{\circ} 38^{\prime} \mathrm{W}, 11-\mathrm{VI}-$ 1987, J.L. Lagares s.n., VAL 56483. Fortanete, Loma del Tajo, 4030' N, 031 'W, 17-VII-1988, G. Mateo 1043, VAL 59652. Javalambre, $40^{\circ} 6^{\prime} \mathrm{N}, 1^{\circ} 0^{\prime} \mathrm{W}$, VII-1975, G. Mateo s.n., VAL 118174. Manzanera a El Toro, $40^{\circ} 3^{\prime} \mathrm{N}, 0^{\circ} 50^{\prime} \mathrm{W}, 22-\mathrm{VI}-1984$, G. Mateo \& al. s.n. VAL 118173. San Agustín, Molino de la Hoz, $40^{\circ} 3^{\prime} \mathrm{N}$, $0^{\circ} 41^{\prime} \mathrm{W}, 21-\mathrm{V}-1999$, F. Gasulla s.n., VAL 108306. Sierra de Albarracín, $40^{\circ} 28^{\prime} \mathrm{N}, 1^{\circ} 30^{\prime} \mathrm{W}, \mathrm{VII}-1965$, E.F. Galiano \& al. s.n., SEV 38286 . Villarluengo, $40^{\circ} 39^{\prime} \mathrm{N}$, $0^{\circ} 31$ ' W, V-1981. A. Aguilella s.n., VAL 10400. Villarroya de los Pinares, Puerto de Villarroya, $40^{\circ} 32$ 'N, $0^{\circ} 40^{\prime} \mathrm{W}, 17-\mathrm{VII}-1977$, B. Casaseca s.n., SALA 19697. VAlENCIA: Ayora, Lomas del Cuemo. Sierra Palomera, $39^{\circ} 4^{\prime} \mathrm{N}, 1^{\circ} 3^{\prime} \mathrm{W}, 20$-VII-1980, J.B. Peris s.n., VAL 118179. Rodenas de Calles, $39^{\circ} 44^{\prime} \mathrm{N}, 0^{\circ} 55^{\prime} \mathrm{W}, \mathrm{VI}-1977$, Mansanet \& Mateo s.n., VAL 11872. Sinarcas, El Picarcho, $39^{\circ} 44^{\prime} \mathrm{N}, 1^{\circ} 14^{\prime} \mathrm{W}, 10$-VI-1993, García Navarro s.n., VAL 92302. Titaguas, Umbría del Ondón, 3952'N, $1^{\circ} 5^{\prime} \mathrm{W}, \mathrm{VI}$, Rojas Clemente s.n., MA 147203. ZaragozA: Moncayo, $41^{\circ} 46^{\prime} \mathrm{N}, 1^{\circ} 50^{\prime} \mathrm{W}, 8$-VII-1906, C. Vicioso s.n., MA 433942. Purujosa, Beraton, $41^{\circ} 41^{\prime} \mathrm{N}, 1^{\circ} 46^{\prime} \mathrm{W}, 21$ VI-1988, D. Gómez \& L Villar s.n., JACA 180088.

MOR, Atlante rifano, $35^{\circ} 0^{\circ} \mathrm{N}, 4^{\circ} 0^{\prime} \mathrm{W}, 16-\mathrm{VI}-1928, R$. Maire s.n., MPU. Atlantis Medii ad Tizi-n-Ourriah sobre Ksiba, $32^{\circ} 30^{\prime} \mathrm{N}, 6^{\circ} 2^{\prime} \mathrm{W}, 27-\mathrm{VI}-1930$, Gattefossé s.n., MPU. Atlas Oriental, 7-VII-1934, R. Maire s.n., MPU. Ksar-es-Souk, cerca de Midkane, $32^{\circ} 32^{\prime} \mathrm{N}, 4^{\circ} 58^{\prime} \mathrm{W}, 8$ VI-1980, A. Charpin \& al. s.n., MA 295124. Meknès, 5 $\mathrm{km}$ Azrou al SE sobre Timahdite. $33^{\circ} 26^{\prime} \mathrm{N}, 5^{\circ} 11^{\prime} \mathrm{W}, 11-$ VI-1980, A. Charpin \& al. s.n., MA 295125. Moyen Atlas, Azrou, ravin de Tioumliline, $33^{\circ} 27^{\prime} \mathrm{N}, 5^{\circ} 14^{\prime} \mathrm{W}$, 20-VI-1923, R. Maire s.n., MPU. Moyen Atlas, forêt d'Ain Raha, $34^{\circ} 34^{\prime}$ N, 5 $21^{\prime}$ W, 2-VII-1939, Maire \& Weiller s.n., MPU. Moyen Atlas, Khenifra. Semni. $33^{\circ} 0^{\circ} \mathrm{N}, 5^{\circ} 40^{\circ} \mathrm{W}, 30-\mathrm{Vl}-1939$, Maire \& Weiller s.n., MPU. Rift Sur, 9-VI-1955, CH. Sauvage s.n., MPU. Meknès, cerca de Aïn Leuh, $33^{\circ} 17^{\prime} \mathrm{N}, 5^{\circ} 23^{\prime} \mathrm{W}, 20$-VI1982. J. Fernández Casas \& al. FC7205. MA 427925. Atlantis Medii, montis Sidi-Ahmer, $33^{\circ} 10^{\circ} \mathrm{N}, 8^{\circ} 28^{\prime}$ W. 8VI-1927, R. Maire s.n.. MPU. 
POR, TrÁs-OS-Montes: Grandais, $41^{\circ} 49^{\prime} \mathrm{N}, 6^{\circ} 49^{\prime} \mathrm{W}$, 19-VI-1968, Pinto da SIlva \& A.N. Teles 8178, LISE 66979.

2. Cephalanthera longifolia (L.) Fritsch in Oesterr. Bot. Z. 38: 81 (1888)

Serapias helleborine var. longifolia $\mathrm{L} .$, Sp. Pl.: 950 (1753) basión.; Serapias longifolia (L.) Huds., Fl. Angl.: 341 (1762). Epipactis longifolia (L.) Wettst. in Oesterr. Bot. Z. 39: 428 (1889). Serapias grandiflora L., Syst. ed. 12: 594 (1767), nom. illeg.; Epipactis pallens $\mathrm{Sw}$. in Neues J. Bot. 1(1): 65 (1805), nom. illeg.; Serapias pallida Wahlenb., Fl. Carpat. Princ.: 291 (1814), nom. illeg.; Cephalanthera pallens (Sw.) Rich., De Orchid. Eur.: 38 (1817), nom. illeg.; Cephalanthera grandiflora Gray, Nat. Arr. Brit. Pl. 2: 210 (1821), nom. illeg.; *Serapias ensifolia Murray., Syst. Veg. ed. 14: 815 (1784), nom. illeg.; $C e$ phalanthera ensifolia Rich., De Orchid. Eur.: 38 (1817), nom. illeg.; Serapias xiphophyllum Ehrh. ex L. f., Suppl. Pl.: 404 (1781). Cephalanthera xiphophyllum (Ehrh. ex L. f.) Rchb. f., Icon. Fl. Germ. Helv. 13-14: 135 (1851). Serapias lonchophyllum L. f., Suppl. Pl.: 405 (1781), nom. illeg.; Serapias nivea Vill., Hist. PI. Dauphiné, 2: 52 (1787), nom. illeg.; $\mathrm{Ce}$ phalanthera longifolia $\mathrm{f}$. angustifolia Maire \& Weiller in Maire, Fl. Afrique N. 6: 359 (1959), nom. illeg.

Cephalanthera angustifolia Simonk., Enum. Fl. Transsilv.: 505 (1887) [n.v.]

Serapias nivea Desf., Fl. Atlant. 2: 321 (1799), nom. illeg. non Vill. (1787)

Cephalanthera xiphophyllum var. latifolia Maire in Bull. Soc. Hist. Nat. Afrique N. 5(6): 239 (1914). Cephalanthera longifolia f. latifolia (Maire) Maire, Fl. Afrique N. 6: 359 (1959. Cephalanthera longifolia var. latifolia (Maire) D. Rivera \& López Vélez, Orquídeas Prov. Albacete: 55 (1987)

Cephalanthera longifolia var. pilosa Harz in Schltdl., Fl. Deutschl. ed. 5, 4: 330 (1896) [n.v.]

Cephalanthera ensifolia var. cesari-gonzalezii Rivas Goday \& Bellot in Anales Jard. Bot. Madrid 5: 437, 439 (1946), nom. nud.; Cephalanthera cesari-gonzalezii Rivas
Goday \& Bellot in Anales Jard. Bot. Madrid 6(2): 198, 210 (1948), nom. nud.

Cephalanthera segobricensis Pau, nom. nud., in sched. (MA 24478)

Ind. loc.: [no indicada expresamente] [neótipo designado por BAUMANN \& al., 1989: 572, Oeder. Fl. Dan. 3(9), tab. 506 (1770)]

Ic.: Ross-Craig, Draw. Brit. Pl. 28, pl. 44 (1971; fig. 6)

Rizoma corto, \pm horizontal, con abundantes raíces fibrosas, fasciculadas. Tallos 17 $60 \mathrm{~cm}$, delgados, flexuosos, estriados, macizos, verdes, glabros. Hojas 7-9 -más 2-4 poco desarrolladas en la base-, de 7-13 $\times 0,7$ $2(3) \mathrm{cm}$, agrupadas, de lanceoladas a lineares -relación anchura/longitud $=0,05-0,33-$, agudas, margen entero, glabras, más largas y estrechas cerca de la inflorescencia. Inflorescencia $5-17(28) \mathrm{cm}, 4-21(28)$ flores sésiles, glabra; bráctea basal 5,5-10,5 × 0,4-1 cm, linear -relación anchura/longitud $=0,04-0,13$, foliácea, aguda, las restantes muy reducidas 0 vestigiales, mucho más cortas que el ovario -relación longitud tercera bráctea/ longitud ovario $=0,15-0,61-$, glabras. Flores 2,5$3,5 \mathrm{~cm}$ de longitud. Sépalos $1,5-2 \times 0,4-$ $0,55 \mathrm{~cm}$, lanceolados, agudos, casi glabros -con pelos glandulíferos dispersos en la cara externa-, blancos. Pétalos laterales 1-1,5 $\times$ $0,4 \mathrm{~cm}$, oblongos, obtusos, glabros, blancos; labelo $0,9-1 \mathrm{~cm}$, menor que los sépalos, glabro; hipoquilo $0,3-0,5 \times 0,8 \mathrm{~cm}$, sacciforme, con dos lóbulos laterales \pm triangulares, obtusos, que encierran el ginostemo, blanquecino; epiquilo 0,4-0,5 × 0,7-0,8 cm -más ancho que largo-, \pm acorazonado, obtuso, blanquecino, con una mancha amarilla en la porción distal, con 5-7 crestas longitudinales de color amarillo anaranjado. Ginostemo 0,8-0,9 cm, \pm cilíndrico, erecto, glabro. Antera $0,25-0,28 \mathrm{~mm}$, oval, cuculada, obtusa; masas polínicas amarillentas. Ovario $0,9-1,8 \mathrm{~cm}$ de longitud, con pelos glandulíferos dispersos. Fruto erecto, oblongo, con 6 costillas. Semillas $0,6-0,75 \times$ 0,14-0,29 mm.

Números cromosomáticos: $2 n=32$ (AvERYANOV \& al., 1985; CAPINERI \& RosSI, 1987), $2 n=34$ (VU \& SOOD, 1986). 


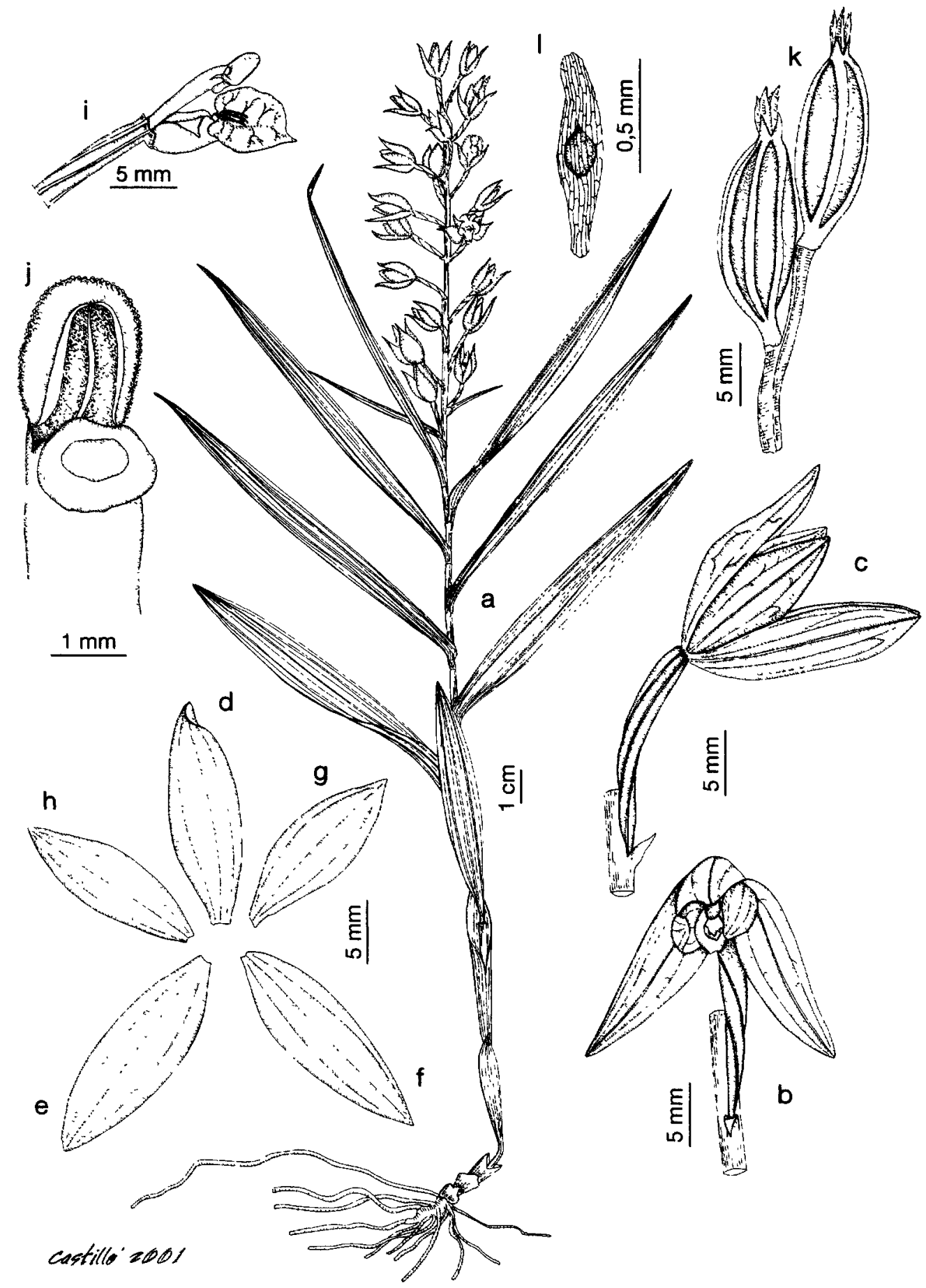

Fig. 6.-Cephalanthera longifolia: a-i, Montemayor del Río, Salamanca (MA 317754); j-1, Ripoll, Gerona (BC 108459): a, hábito; $b$, flor, vista frontal; $c$, flor, vista lateral; d-f, sépalos; g,h, pétalos; i. ginostemo y labelo; j, detalle del ginostemo con las anteras y el disco estigmático; $\mathbf{k}$, cápsula; 1 , semilla. 
Hábitat: Bosques caducifolios, encinares y pinares; en todo tipo de suelos; 0-1700(1900) $\mathrm{m}$.

Floración: (III)IV-VII(X).

Área de distribución: En la mayor parte de Europa, SW de Asia -alcanza el Pamir y el Himalaya central-, Cáucaso, Anatolia y NW de África. Toda la Península e Islas Baleares.

Es la especie de más amplia distribución en el área de este estudio. Únicamente falta en el $S$ de Portugal y en amplias zonas de la Meseta norte. En las Islas Baleares solo se conoce de Mallorca. En el N de África se encuentra en diversos puntos de Marruecos y Argelia (MAIRE, 1959), así como en una localidad tunecina (Cú́Nod, 1954) (fig. 7).

No hemos podido estudiar material de Andorra (cf. LOSA \& MONTSERRAT, 1950: 152), y de las siguientes provincias: Almería (cf. PALLARÉS NAVARRO, 1999: 42-43), Asturias (cf. NAVARRO ANDRÉS \& DÍAZ GONZÁlEZ, 1977: 49), Palencia (cf. ORIA DE RUEDA \& al., 1996: 157) y Vizcaya (cf. AsEGINOLAZA \& al., 1985: 1096).

\section{Material estudiado}

ALG, Djurdjura, forêt des Aït Ouaban, $36^{\circ} 26^{\prime} \mathrm{N}$. $4^{\circ} 13^{\prime} \mathrm{E}, 21$-VII-1936, Saccardy s.n., MPU. Entre Ben Aknoun y Crescia, $36^{\circ} 46^{\prime} \mathrm{N}, 3^{\circ} 1$ 'E, J.A. Battandier s.n., MPU. Oued Djer, 36 36'N, $2^{\circ} 44^{\prime}$ E, V-1978, J.A. Battandier s.n., MPU.

SPA, ÁlaVA: Fontecha, $42^{\circ} 45^{\circ} \mathrm{N}, 3^{\circ} 1 \mathrm{~W}, 22-\mathrm{V}$ 1985, P.M. Uribe-Echebarría s.n., MA 500020. Lantarón, Fontecha, $42^{\circ} 45^{\prime} \mathrm{N}, 3^{\circ} 1$ 'W, 19-V-1984, J.A. Alejandre s.n., MA 400661 . Sierra de Cantabria, $42^{\circ} 36^{\prime} \mathrm{N}$, $2^{\circ} 35^{\prime}$ W, 15-VI-1984, I. Aizpuru \& Catalán s.n., ARAN 32516. Sobrón, Salinas de Añana, $42^{\circ} 46^{\prime} \mathrm{N}, 3^{\circ} 7^{\prime} \mathrm{W}, 7-\mathrm{V}$ 1988, J. Elorza s.n., ARAN 55241. ALBACETE: Canaleja a Viveros, $38^{\circ} 42^{\prime} \mathrm{N}, 2^{\circ} 33^{\prime} \mathrm{W}, 26-\mathrm{IV}-1987, F$. Esteso \& Peris s.n. VAL 118180. Sierra de Alcaraz, $38^{\circ} 35^{\circ} \mathrm{N}$, $2^{\circ} 24^{\prime}$ W, 16-V-1986, Lansac \& Nieto Feliner I268GN, MA 393854. AliCANTE: Sierra de Salinas, Villena, $38^{\circ} 38^{\prime}$ N, $0^{\circ} 51^{\prime} \mathrm{W}, 26-\mathrm{V}-1956$, A. Rigual s.n., MA 373109. Ávila: Barraco, Puenteladrillo, $40^{\circ} 29^{\prime} \mathrm{N}$, 43' W, 29-V-1988, F. Gómez Manzaneque s.n., MA 588003. Candeleda, $40^{\circ} 15^{\prime} \mathrm{N}, 5^{\circ} 15^{\prime} \mathrm{W}$, Luceño \& Vargas 1082PV, MA 321752. Mombeltrán, $40^{\circ} 16^{\prime} \mathrm{N}, 5^{\circ} 1$ 'W, 19 IV-1984, P. Garín s.n., ARAN 32517. BADAJOZ: Sierra de Valdecaballeros, $39^{\circ} 15^{\prime} \mathrm{N}, 5^{\circ} 11^{\prime} \mathrm{W}, 21$-IV-1973, J.L. Pérez Chiscano s.n., VAL 118182. Valdecaballeros, $39^{\circ} 15^{\prime} \mathrm{N}, 5^{\circ} 11^{\prime} \mathrm{W}, 21-\mathrm{V}-1973$, J.L. Pérez Chiscano s.n., SALA 5908. Baleares: Ibiza, Canal d'en Sastre, St. Joan, 10-IV-1999, N. Torres s.n. (MA, fotocopia). Mallorca, Escorca al Gorg Blau, 3949'N, $2^{\circ} 51^{\prime} \mathrm{E}, 12-\mathrm{V}$ -

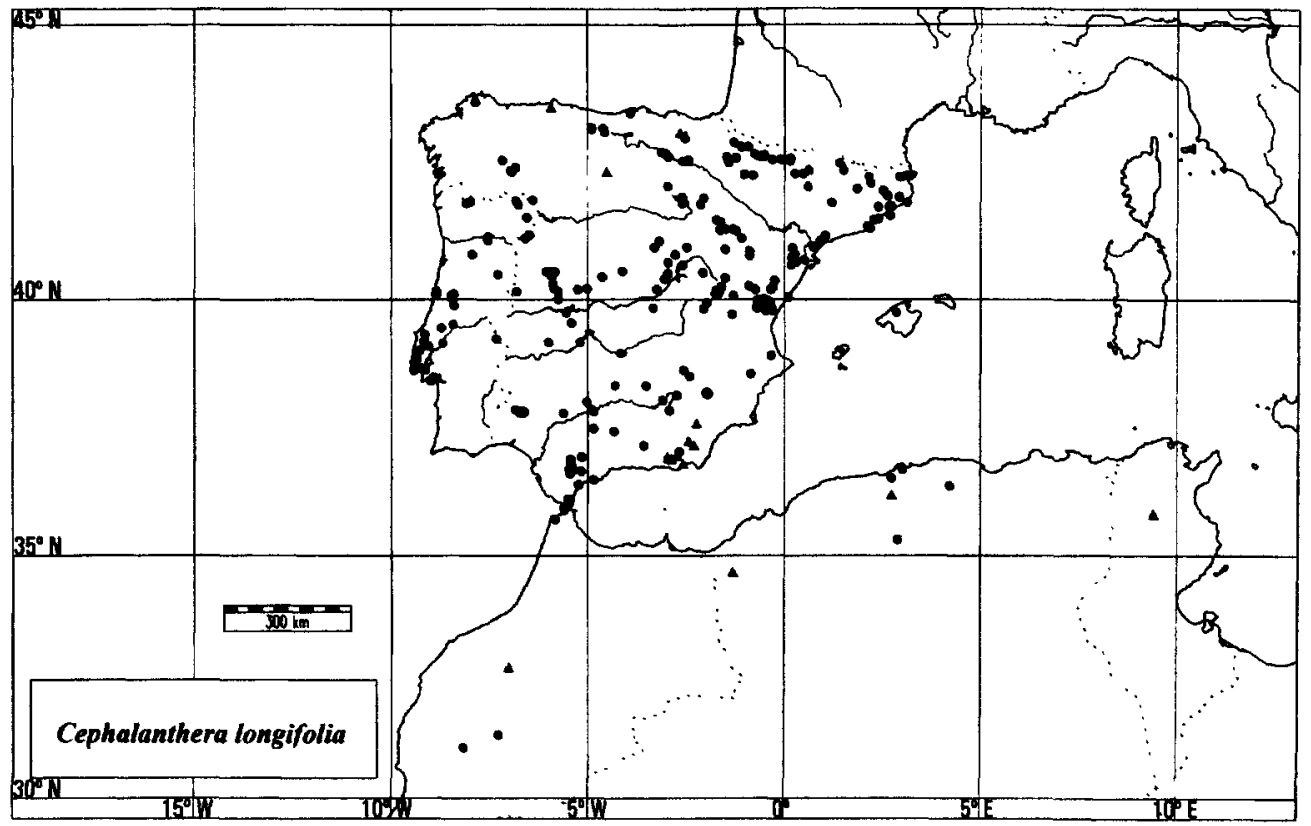

Fig. 7.-Distribución de Cephalanthera longifolia en la Península Ibérica y N de África (•, material estudiado; ^, citas bibliograficas). 
1947, Palau Ferrer s.n., LISE 31260. BARCELONA: Alt Berguedà, la Clusa. $42^{\circ} 6^{\prime} \mathrm{N}, 1^{\circ} 51^{\circ} \mathrm{E}, 7$-VI-1975, A. Rosell s.n., BC 621639. Coto de las Aduanas, Tibidabo, $41^{\circ} 25^{\prime} \mathrm{N}, 2^{\circ} 7^{\prime} \mathrm{E}, 1911$, Basilio s.n., BC 60097. Massif del Tibidabo, $41^{\circ} 25^{\prime}$ N, $2^{\circ} 7^{\prime}$ E, 30-III-1920, F. Sennen s.n., MA 24469. Montnegre, 41 ${ }^{\circ} 57^{\prime} \mathrm{N}, 2^{\circ} 56^{\prime} \mathrm{E}, 19-\mathrm{IV}-1949$, P. Montserrat \& M. Losa s.n., JACA 10549 A. Montseny, entre Sant Elies i El Terrer. $41^{\circ} 46^{\prime} \mathrm{N}, 2^{\circ} 24^{\prime} \mathrm{E}, 31$ III-1995, A. Salvador \& J. Vicens s.n., BCF 51220. Muntanyes d'Argentona, Brolla de l'Abril, $41^{\circ} 33^{\prime} \mathrm{N}, 2^{\circ} 24^{\prime} \mathrm{E}$, 28-IV-1946, P. Montserrat s.n., BC 620595. Pineda, Turó de la Guàrdia, $41^{\circ} 37^{\prime} \mathrm{N}, 2^{\circ} 42^{\prime} \mathrm{E}, 17-\mathrm{IV}-1946$, P. Montserrat s.n., BC 620594. Rabassa, vertiente norte del Tibidabo, $41^{\circ} 25^{\prime}$ N, $2^{\circ} 7$ 'E, 22-V-1936, Rocha s.n., MA 345608. Sant Boi de Llobregat, $41^{\circ} 23^{\prime} N, 2^{\circ} 11^{\prime} E, 2-$ IV-1916, P. Font Quer s.n., BC 60132. Vallromanas. Maresme. $41^{\circ} 32^{\prime} \mathrm{N}, 2^{\circ} 17^{\prime} \mathrm{E}, 21$-IV-1921, J.M. Barnadés s.n., BC 603737. Burgos: Monte Herrera, $42^{\circ} 41^{\prime} \mathrm{N}$, 257'W, V-1928, M. Losa s.n., BCF 3771. CÁCERES: Bajada del Puerto de Santa Clara a San Martín de Trevejo, $40^{\circ} 13^{\prime} \mathrm{N}, 6^{\circ} 48^{\prime} \mathrm{W}, 7-\mathrm{V}-1978$, A. Valdés Franzi s.n., SALA 21132. Castañar de Ibor, 39 $37^{\prime} N$, 5 $25^{\prime} \mathrm{W}, 16-$ IV-1967, M. Ladero s.n., MA 588466. Hervás, $40^{\circ} 16^{\prime} \mathrm{N}$, 5०51'W, 17-V-1982, F. Amich \& F. Herrero s.n., SALA 34978. Jaraíz de la Vera, $40^{\circ} 4^{\prime} \mathrm{N}, 5^{\circ} 45^{\prime} \mathrm{W}, 16-I V-1987$, A. Amor s.n., SALA 15727. Jerte, $40^{\circ} 13^{\prime}$ N, $5^{\circ} 45^{\prime} \mathrm{W}, 4$ V-1990, M.A. Carrasco \& S. Pajarón s.n., MA 520669. Río Ibor, 3949' N, $5^{\circ} 33^{\prime} \mathrm{W}, 10-\mathrm{V}-1980$, Aquedo, Ávila \& Viera s.n., MA 453981. Subida al Puerto de Robledillo, $39^{\circ} 15^{\circ} \mathrm{N}, 5^{\circ} 59^{\prime} \mathrm{W}, 11-\mathrm{VI}-1978$, A. Valdés Franzi s.n., SALA 21131. CÁDIZ: Algeciras, Sierra de la Luna, $36^{\circ} 7^{\prime}$ N, 527' W, 29-III-1979, B. Cabezudo \& al. 164/79, SEV 67659. Algodonales, Sierra de Lijar, $36^{\circ} 55^{\prime} \mathrm{N}$, 5'26'W, 20-V-1981, A. Aparicio \& C. Romero 51/81, SEV 71481. Benamahoma, $36^{\circ} 46^{\prime} \mathrm{N}, 5^{\circ} 28^{\prime} \mathrm{W}, 29$-IV1984, A. Aguilella s.n., VAL 10510. Grazalema, camino del Pinsapar desde Zahara, $36^{\circ} 46^{\prime} \mathrm{N}, 5^{\circ} 22^{\prime} \mathrm{W}$. 18-IV1982, J. Arroyo s.n., SEV 84072. Los Barrios, valle del Arroyo de la Hoya, 36 $11^{\prime} \mathrm{N}, 5^{\circ} 30^{\prime} \mathrm{W}, 20$-III-1981, J. Arroyo \& al. J1864/81, SEV 68714. Montes propios de Jerez, entre Ubrique y Puerto de Galiz, $36^{\circ} 41^{\prime} \mathrm{N}, 5^{\circ} 27^{\prime} \mathrm{W}$, 21-III-1980, J. Ubera \& B. Valdés 332/80, SEV 67658. Tarifa. Sierra de Ojén, $36^{\circ} 1^{\prime} N, 5^{\circ} 36^{\prime} \mathrm{W}, 16-I V-1974$, S. Talavera \& B. Valdés s.n., SEV 67656. Tarifa, Sierra de Saladavieja, 36 ${ }^{\circ} 1^{\prime} \mathrm{N}, 5^{\circ} 36^{\prime} \mathrm{W}, 30-\mathrm{III}-1980, F$. Amor \& J. Arroyo s.n., SEV 67484. Zahara, Sierra Margarita, 36 $6^{\circ} 0^{\prime} \mathrm{N}, 5^{\circ} 24^{\prime} \mathrm{W}, 28$-III-1983, A. Aparicio \& S. Silvestre A.A.943, SEV 161646. CANTABRIA: Cabezón de Liébana, la Barga del Caballo, 43ㅇ' ${ }^{\prime}, 4^{\circ} 34^{\prime} \mathrm{W}, 18-\mathrm{V}-1994$, G. Moreno Moral \& Ó. Sánchez Pedraja s.n. (herb. Sánchez Pedraja-3285. Cillórigo de Liébana, monte Osina, $43^{\circ} 12^{\prime} \mathrm{N}, 4^{\circ} 36^{\prime} \mathrm{W}, 19-\mathrm{VII}-2000$, Aldasoro \& al. MMO305 (herb. Sánchez Pedraja-9321. Invernales de Lebeña, Cillórigo-Castro, $43^{\circ} 12^{\prime} \mathrm{N}, 4^{\circ} 36^{\prime} \mathrm{W}, 25-\mathrm{IV}-1991$, C. Aedo s.n., MA 623393. Liencres, $43^{\circ} 28^{\prime} \mathrm{N}, 3^{\circ} 55^{\prime} \mathrm{W}$, 15-V-1985, C. Aedo s.n., MA 598358. CASTELlóN: Ahín, $39^{\circ} 54^{\prime} \mathrm{N}, 0^{\circ} 20^{\prime} \mathrm{W}, 20-\mathrm{V}-1984, G$. Mateo \& A. Aguilella s.n., VAL 51440. Algimia de Almonacid, bco. del Agua Negra, 39 $55^{\prime} \mathrm{N}, 0^{\circ} 26^{\prime} \mathrm{W}, 3-\mathrm{V}-2000, T$. Castello 105JNT, VAL 43045. Alt Maestrat, Sant Joan de Penyagolosa, $40^{\circ} 15^{\prime} \mathrm{N}, 0^{\circ} 21^{\prime} \mathrm{W}, 25-\mathrm{V}-1968$, J. Vigo \& A. Anglada s.n., BC 599764. Alt Palància, Pina de Montalgrao, $40^{\circ} 1^{\prime} \mathrm{N}$, $0^{\circ} 39^{\prime} \mathrm{W}, 28-\mathrm{V}-1989, J$. Riera 731JRV, VAL 21535. Ayodar, $40^{\circ} 0^{\prime} \mathrm{N}, 0^{\circ} 22^{\prime} \mathrm{W}, 4-\mathrm{V}-1996, C$. Maciän s.n., VAL 99075. Bejís, 3954'N, 042'W, 6-VI-1984, A. Aguilella s.n., VAL 5992. Benicasim, Desierto de las Palmas. 406'N, 06'E, 13-IV-1986, G. Mateo s.n., VAL 53719. Chovar, $39^{\circ} 51^{\prime} \mathrm{N}, 0^{\circ} 19^{\prime} \mathrm{W}, 16-\mathrm{IV}-2000$, J. Andrés \& al. 19JNT, VAL 42982. Matet, bco. de Camperí, $39^{\circ} 56^{\prime} \mathrm{N}$, $0^{\circ} 28^{\prime} \mathrm{W}, 29-\mathrm{IV}-2000, J$. Andrés \& al. 22JNT. VAL 42990. Montanejos, $40^{\circ} 4^{\prime} \mathrm{N}, 0^{\circ} 31^{\prime} \mathrm{W}, 10$-VI-1992, L. Marín s.n., VAL 87036. Montes de Fredes, $40^{\circ} 42^{\prime} \mathrm{N}$, $0^{\circ} 10^{\prime} \mathrm{E}, \mathrm{VI}-1979$, Mansanet \& Mateo s.n., VAL 46127. Pavias, bco. de Pavías. $39^{\circ} 58^{\prime} \mathrm{N}, 0^{\circ} 29^{\prime} \mathrm{W}, 30$-IV-2000, $J$. Andrés \& al. 29JNT. VAL 43001. Segorbe. Montemayor, 39 $51^{\prime} \mathrm{N}, 0^{\circ} 29^{\prime} \mathrm{W}, \mathrm{V}-1912$, C. Pau s.n., MA 24478. Sierra de Espadán. 3957'N, $0^{\circ} 25^{\prime} \mathrm{W}, 19-\mathrm{V}-1887$. C. Pau s.n., MA 24468. Torralba del Pinar, $39^{\circ} 59^{\prime} \mathrm{N}, 0^{\circ} 26^{\circ} \mathrm{W}$, 15-V-1982, G. Mateo \& F. Boisset s.n., VAL 48226. Villafranca del Cid, Pinar de Coder, $40^{\circ} 25^{\prime} \mathrm{N}, 0^{\circ} 15^{\prime} \mathrm{W}, 4-$ VI-1992, G. Mateo \& al. 3334CF, VAL 89857. Vistabella del Maestrazgo, $40^{\circ} 18^{\prime} \mathrm{N}, 0^{\circ} 17^{\prime} \mathrm{W}, \mathrm{VI}-1980$, Mansanet \& Mateo s.n., VAL 46681. CiUdad REAL: Fuencaliente, $38^{\circ} 24^{\prime} \mathrm{N}, 4^{\circ} 18^{\prime} \mathrm{W}, 22-$-III-1997, $R$. García Río s.n., MA 596777. Piedrabuena. junto al río Bullaque, $39^{\circ} 2 ' \mathrm{~N}$, $4^{\circ} 10^{\prime}$ W, 1-V-1991. J.C. Garrido \& al. s.n., MA 594202. CiUdAD Real-JAÉN: Sierra Morena, Despeñaperros, $38^{\circ} 24^{\prime} \mathrm{N}, 3^{\circ} 30^{\prime} \mathrm{W}, 30-\mathrm{IV}-1933$, J. Cuatrecasas s.n., MA 24467. CóRdoBA: Carretera de Villaviciosa de Córdoba a Posadas, km 40, 38 ${ }^{\circ} \mathrm{N}, 5^{\circ} 1^{\prime} \mathrm{W}, 19-\mathrm{IV}-1981$, P. Fernández \& al. 2000/81, SEV 71230. Entre Santaella y la Mar-

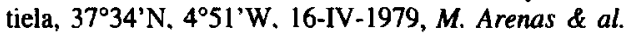
s.n., SEV 71233. Entre Trassierra y el rio Guadalmellato, 37'56'N, 453'W, 5-V-1982, M.J. Diez \& I. Fernández s.n., SEV 88271. Sierra de Cabra, $37^{\circ} 30^{\prime} N, 4^{\circ} 20^{\prime} \mathrm{W}, 5-$ V-1918, C. Vicioso s.n., MA 24463. Sierra de Córdoba, junto a Assuan, $37^{\circ} 54^{\prime}$ N. $4^{\circ} 51^{\prime} \mathrm{W}, 31-\mathrm{III}-1978, J . A . D e$ vesa s.n., SALA 19568. CuENCA: Aliaguilla, Pico Mazmorra, $39^{\circ} 47^{\prime} \mathrm{N}, 1^{\circ} 20^{\prime} \mathrm{W}, \mathrm{V}-1978, G$. Mateo s.n., VAL 118167. Beteta, valle del Tajo, estrecho del Horcajo, $40^{\circ} 34^{\prime} \mathrm{N}, 2^{\circ} 4^{\prime} \mathrm{W}, 28-\mathrm{V}-1995$, G. Mateo \& al. $10503 \mathrm{GM}$, VAL 93246 . Hoyo de las Casas, Hoz de Beteta, $40^{\circ} 34^{\prime} \mathrm{N}$, $2^{\circ} 4$ 'W, 12-V-1933, A. Caballero s.n., MA 24459. Hoz Chiquilla, monte de los Palancares, $40^{\circ} 0^{\prime} \mathrm{N}, 1^{\circ} 58^{\prime} \mathrm{W}, 29$ IV-1995, G. Moreno Valdeolivas s.n., VAL 92951. Laguna del Marquesado, $40^{\circ} 11^{\prime} \mathrm{N}, 1^{\circ} 40^{\prime} \mathrm{W}, 25-\mathrm{V}-1974$, A. González \& G. López s.n., MA 392991. Los Cadorzos, 39 $53^{\prime} \mathrm{N}, 2^{\circ} 3^{\prime} \mathrm{W}, 24-\mathrm{V}-1956$, C. Vicioso s.n., MA 171699. Sierra de Valdemeca, $40^{\circ} 10^{\prime} \mathrm{N}, 1^{\circ} 45^{\prime} \mathrm{W}, 23-$ VIII-1974, G. López s.n., MA 393030. GERONA: Cabanas, 42 $19^{\prime}{ }^{\prime}, 2^{\circ} 58^{\prime} E, 28-I V-1907, F$. Sennen s.n., BC 837517. Empalme, $41^{\circ} 46^{\prime} \mathrm{N}, 2^{\circ} 41^{\prime} \mathrm{E}, 11-\mathrm{IV}-1948$, Casellas s.n., BCF 3763. Entre puerto de Condreu y el puerto de Can Bach, 42 ${ }^{\circ}$ 'N, $2^{\circ} 31^{\prime}$ E, 3-VI-1981, J.A. Devesa \& al. s.n., SEV 71231. La Sellera, $41^{\circ} 58^{\prime} \mathrm{N}, 2^{\circ} 38^{\prime} \mathrm{E}$, V1908, J. Codina s.n., BCF 51223. Martorell de la Selva y Tordera, $41^{\circ} 47^{\prime} \mathrm{N}, 2^{\circ} 44^{\prime} \mathrm{E}, 10-\mathrm{IV}-1949, F . C l$. \& al. s.n., BC 108735. Palamós, $41^{\circ} 51^{\prime} \mathrm{N}, 3^{\circ} 8^{\prime} \mathrm{E}, 11$-IV-1949, P. Montserrat s.n., JACA 16949 A. Pont de Molins, $42^{\circ} 19^{\prime}$ N, 2 ${ }^{\circ} 56^{\prime}$ E, 8-VIII-1907, F. Sennen s.n., BC 837524 B. Port de la Selva, Cala Taballera, $42^{\circ} 20^{\prime} \mathrm{N}$, $3^{\circ} 12^{\prime} \mathrm{E}$, IV-1957, Losa \& Casas s.n., BCF 51222. Ripoll, $42^{\circ} 12^{\prime} \mathrm{N}, 2^{\circ} 12^{\prime} \mathrm{E}, 2-\mathrm{IX}-1945$, A. Bolòs \& O. Bolòs s.n., BC 108459. Vall de Ribes, sobre Can Perramon de Baix, 
$42^{\circ} 19^{\prime} \mathrm{N}, 2^{\circ} 9^{\prime} \mathrm{E}, 1-\mathrm{VI}-1969$, J. Vigo s.n., BC 604141 . Vilajuïga, $42^{\circ} 20^{\prime} \mathrm{N}, 3^{\circ} 6^{\prime} \mathrm{E}, 24-\mathrm{IV}-1908$, F. Sennen s.n., BC 837518. GRANADA: Sierra de Alfacar, $37^{\circ} 14^{\prime} \mathrm{N}, 3^{\circ} 34^{\prime} \mathrm{W}$, 29-V-1978, J.A. Devesa \& al. 3997/78, SEV 61150. Guadalajara: Alcolea del Pinar, $41^{\circ} 2^{\prime} \mathrm{N}, 2^{\circ} 28^{\prime} \mathrm{W}, 8$ VI-1971. Carballal s.n., SALA 33226. Almadrones, $40^{\circ} 54^{\prime} \mathrm{N}, 2^{\circ} 46^{\prime} \mathrm{W}, 12-\mathrm{V}-1962$, E.F. Galiano s.n., SEV 6873. Embalse del Vado, $41^{\circ} 2^{\prime} \mathrm{N}, 3^{\circ} 18^{\prime} \mathrm{W}, 20$-IV-1987, F. Garín s.n.. ARAN 32511. Hontoba, Monte Carabo, $40^{\circ} 27^{\prime} \mathrm{N}, 3^{\circ}{ }^{\prime}$ 'W, 11-V-1969, Bellot \& Ron s.n., MA 95272. La Fuensaviñán, $40^{\circ} 45^{\prime} \mathrm{N}, 2^{\circ} 57^{\prime} \mathrm{W}, 26-\mathrm{V}-1994$, $J$. Castillo \& al. 7289MV, MA 543892. La Huerce, $41^{\circ} 9^{\prime} \mathrm{N}, 3^{\circ} 10^{\prime} \mathrm{W}, 2-\mathrm{VI}-1987, F$. Lamata s.n., MA 587677. Tendilla a Alhóndiga, $40^{\circ} 33^{\prime} \mathrm{N}, 2^{\circ} 57^{\circ} \mathrm{W}, 19-\mathrm{V}-$ 1974, G. López 1239GF, MA 392996. Trillo, 40²'N, $2^{\circ} 35$ 'W, 13-V-1970, Bellot \& Ron s.n., MA 95263. GúpúzCoA: Escoriaza, $43^{\circ} 1^{\prime} \mathrm{N}, 2^{\circ} 31^{\prime} \mathrm{W}$, Gredilla s.n., MA 585202. Huelva: Aguafría, Sierra de Aracena, $37^{\circ} 53^{\prime} \mathrm{N}, 6^{\circ} 44^{\prime} \mathrm{W}, 9-\mathrm{IV}-1979$, J. Rivera 3910R, MA 295392. Alajar, Sierra de Aracena, $37^{\circ} 52^{\prime} \mathrm{N}, 6^{\circ} 39^{\prime} \mathrm{W}, 7-$ IV-1978, J. Rivera 469R, SEV 50230. Castaño de Robledo, 37 $54^{\prime}$ N, $6^{\circ} 42^{\prime}$ W, 3-V-1984, Garcia, P.L. Ortiz \& J.L. Pastor s.n., SEV 161639. Fuenteheridos, Sierra de Aracena, $37^{\circ} 54^{\circ} \mathrm{N}, 6^{\circ} 39^{\prime} \mathrm{W}, 25$-IV-1978, J. Rivera $1239 R$, SEV 50231. Galaroza, $37^{\circ} 55^{\prime} \mathrm{N} .6^{\circ} 42^{\prime} \mathrm{W}, 11$-IV1980, B. Casaseca \& al. s.n., MA 487194. Linares de la Sierra, $37^{\circ} 53^{\prime} \mathrm{N}, 6^{\circ} 37^{\prime} \mathrm{W}, 16-$ III-1982, M.J. Diez \& P. Murillo s.n., SEV 88273. Los Marines, $37^{\circ} 54^{\prime} \mathrm{N}$, $6^{\circ} 37^{\prime} \mathrm{W}, 27-I V-1981$, F. García \& al. s.n., SEV 71479. Sierra de Aracena, cruce Valdelarco con Navahermosa, $37^{\circ} 56^{\prime} \mathrm{N}, 6^{\circ} 50^{\prime} \mathrm{W}, 25-\mathrm{IV}-1978$, J. Rivera $1172 R$, SEV 50233. HuesCA: Agüero, $42^{\circ} 21^{\circ} \mathrm{N}, 0^{\circ} 48^{\prime} \mathrm{W}, 3-\mathrm{V}-1980$, P. Montserrat \& al. s.n., JACA 28980 . Araguiés del Puerto, Lavati, $42^{\circ} 42^{\prime} \mathrm{N}, 0^{\circ} 40^{\prime} \mathrm{W}, 13-\mathrm{VIII}-1969$, $P$. Montserrat s.n., JACA 688069. Biescas, bco. del Puerto, $42^{\circ} 38^{\prime} \mathrm{N}, 0^{\circ} 19^{\prime} \mathrm{W}, 22-\mathrm{VI}-1978$, L. Villar s.n., JACA 126678. Bosque de la Solana, cerca de Canfranc, $42^{\circ} 43^{\prime} \mathrm{N}, 0^{\circ} 31^{\prime} \mathrm{W}, 24-\mathrm{VI}-1928$, s.n., MPU 12428. Egea de Turbón, $42^{\circ} 22^{\prime} \mathrm{N}, 0^{\circ} 28^{\prime} \mathrm{E}, 31-\mathrm{V}-1992$, J.A. Sesé s.n. JACA 73892. Escuain, $42^{\circ} 36^{\prime} \mathrm{N}, 0^{\circ} 8^{\circ} \mathrm{E}, 8$-VII-1990, D. Gómez s.n., JACA 165590 . Espierba, Bielsa, 42 ${ }^{\circ} 39^{\prime} \mathrm{N}$, 09'E, 20-VI-1991, L. Villar \& al. s.n., JACA 95591. Hecho, Selva de Oza, 42 $2^{\circ} 44^{\prime} \mathrm{N}, 0^{\circ} 45^{\prime} \mathrm{W}, 17$-VII-1973, L Villar s.n., JACA 10030273. La Fueva, Rañín, 42 $22^{\prime} \mathrm{N}$, $0^{\circ} 16$ 'E, 10-VII-1992, J.V. Ferrández s.n., JACA 479392. Torla, puente Los Navarros, $42^{\circ} 38^{\prime} \mathrm{N}, 0^{\circ} 6^{\prime} \mathrm{W}, 27-\mathrm{VI}-$ 1978, P. Montserrat \& J.M. Montserrat s.n., JACA 135678. Viacamp y Litera, $42^{\circ} 8^{\prime} \mathrm{N}, 0^{\circ} 36^{\prime} \mathrm{E}, 16$-VIII1996. J.V. Ferrández s.n. JACA 371396. Villanúa, $42^{\circ} 41^{\prime} \mathrm{N}, 0^{\circ} 32^{\prime} \mathrm{W}, 9-\mathrm{VI}-1973$, J. Puigdefábregas s.n., JACA 153973. JAÉN: Despeñaperros. Valdeazores, $38^{\circ} 24^{\prime} \mathrm{N}, 3^{\circ} 30^{\prime} \mathrm{W}, 28-\mathrm{V}-1967$, P. Montserrat \& F. García Novo s.n., JACA 177867 . Hornos, $38^{\circ} 13^{\prime}$ N, $2^{\circ} 43^{\prime}$ W, 10-IV-1983, C. Cebolla \& al s.n., MA 462029. Pontones, cerro del Espartal, $37^{\circ} 7^{\circ} \mathrm{N}, 2^{\circ} 40^{\prime} \mathrm{W}, 7$-V -1975 , González Rebollar \& al. s.n., MA 481209. Sierra de Cazorla, río Borosa, $37^{\circ} 55^{\prime} \mathrm{N}, 2^{\circ} 55^{\prime} \mathrm{W}, 19-\mathrm{V}-1982, P$. Garcia Murillo s.n., SEV 161645. Villacarrillo, Sierra de las Villas, $38^{\circ} 7^{\prime} \mathrm{N}, 3^{\circ} 5 \mathrm{~W}, 3-\mathrm{V}-1985$, C. Soriano s.n., MA 462028. La CoRuÑa: Ortigueira, praia de Cabalar, $43^{\circ} 40^{\prime} \mathrm{N}, 7^{\circ} 51^{\prime} \mathrm{W}, 24-1 \mathrm{~V}-1993$, J.M. Sánchez s.n., SANT. La Rioja: Mansilla de la Sierra, 42 ${ }^{\circ}$ 'N, $2^{\circ} 57^{\prime} \mathrm{W}$,
28-V-1988, J. Arizaleta \& al. 3042JP, MA 438751. LeÓN: Caín, $43^{\circ} 13^{\prime} N, 4^{\circ} 54^{\prime} W, 14-\mathrm{V}-1978, C$. Garcia González s.n., JACA 129685. Corona, 4311'N, 4⒌'W, 3-VI-1979, C. García González s.n., JACA 519285. Peñarrubia, $42^{\circ} 30^{\circ} \mathrm{N}, 6^{\circ} 49^{\prime} \mathrm{W}, 10-\mathrm{IV}-1990$, J. Amigo \& $M$. Romero s.n., SANT 26304. Lugo: Seoane, camín Rial, $42^{\circ} 38^{\prime} \mathrm{N}, 7^{\circ} 9^{\prime} \mathrm{W}, 4-\mathrm{VI}-1981$, J. Izco \& al. s.n., SANT 14704. Lérida: Alta Ribagorça, pr. Senyiu, $42^{\circ} 26^{\prime} \mathrm{N}$, $0^{\circ} 37^{\prime}$ E, 7-VI-1987, J. Carreras \& E. Carrillo s.n., BCN 68. Castelló de Tor, $42^{\circ} 34^{\prime} \mathrm{N}, 1^{\circ} 23^{\prime} 59^{\prime \prime} \mathrm{E}, 14-\mathrm{VI}-1984$, J.M. Carrillo \& Ninot s.n,, BCN 70. Vall Fosca, Molinos, 4226' N, $1^{\circ} 30^{\prime}$ E, 14-VI-1985, L. Pineda s.n., BCN 78. MADRID: Dehesa de Carabaña, $40^{\circ} 15^{\prime} \mathrm{N}, 3^{\circ} 14^{\prime} \mathrm{W}, 18 \mathrm{~V}$ 1900, Reyes s.n., MA 24458. El Escorial, $40^{\circ} 35^{\prime} \mathrm{N}$, $4^{\circ} 7$ 'W, 15-V-1963, P. Montserrat s.n., JACA 210463. MálaGA: Ronda. $36^{\circ} 44^{\prime} \mathrm{N}, 5^{\circ} 10^{\circ} \mathrm{W}, 25$-IV-1932, L. Ceballos s.n., MA 24464. Sierra Bermeja de Ojén, entre Ojén y Monda, 36² $34^{\prime}$ N, $4^{\circ} 51^{\prime}$ W, 20-IV-1973, S. Talavera \& B. Valdés s.n., SEV 61756. Torre Bermeja, $36^{\circ} 28^{\prime} \mathrm{N}$. $5^{\circ} 14^{\prime} \mathrm{W}, 20$-III, Rodríguez s.n., MA 24465. MURCIA: Sierra de los Álamos, $38^{\circ} 10^{\prime} \mathrm{N}, 01^{\circ} 56^{\prime} \mathrm{W}, 3-\mathrm{V}$ 2002. C. Aedo 7572 (MA). NaVARRA: Ezprogi, Sabaiza, $42^{\circ} 35^{\prime} \mathrm{N}, 1^{\circ} 25^{\prime} \mathrm{W}, 10-\mathrm{V}-1987$. I. Aizpuru \& Catalán s.n., ARAN 32510. Garralda, 42 $57^{\prime} \mathrm{N}, 1^{\circ} 17^{\prime} \mathrm{W}, 14-\mathrm{V}-1988$, I. Aizpuru \& Catalán s.n., ARAN 32508, Ibargoiti, Idocín, barranco Ubarka, $42^{\circ} 41^{\prime} \mathrm{N}, 1^{\circ} 28^{\prime} \mathrm{W}, 25-\mathrm{V}-1987$, I. Aizpuru \& Catalán s.n., ARAN 32509. Isaba, bco. de Belabarte, $\quad 42^{\circ} 52^{\prime} \mathrm{N}, \quad 0^{\circ} 55^{\prime} \mathrm{W}$, 20-VI-1975, L. Villar s.n., JACA 10025875. Marañón, $42^{\circ} 38^{\prime}$ N, $2^{\circ} 26^{\prime} \mathrm{W}, 12-\mathrm{VI}-1981$, Puy Isaba s.n., ARAN 48642 A. Meano, $42^{\circ} 36^{\prime} \mathrm{N}, 2^{\circ} 28^{\prime} \mathrm{W}, 19-\mathrm{V}-1982, P u y$ Isaba s.n., ARAN 48644. Oronz, 42 $2^{\circ} 52^{\prime} \mathrm{N}, 1^{\circ} 6$ 'W, 1 -VI1986, I. Aizpuru \& Catalán s.n., ARAN 32512. Romanzado. Foz de Arbaiun, $42^{\circ} 40^{\prime} \mathrm{N}, 1^{\circ} 14^{\prime} \mathrm{W}, 12-\mathrm{V}-1985$, 1. Aizpuru \& al. s.n., ARAN 32514. ORENSE: Rubiá, Cobas, $42^{\circ} 26^{\circ} \mathrm{N}, 6^{\circ} 57^{\prime} \mathrm{W}, 30-\mathrm{IV}-1992$, J. Amigo \& $M$. Romero s.n., SANT 27347. Sobradelo hacia Casaio, $42^{\circ} 25^{\prime} \mathrm{N}, 6^{\circ} 55^{\prime} \mathrm{W}, 27-\mathrm{IV}-1981$, J. Izco \& Santiago s.n. SANT 16092. PoNTEvedra: Sanxenxo, monte de Cabicastro, $42^{\circ} 24^{\prime} \mathrm{N}, 8^{\circ} 49^{\prime} \mathrm{W}, 9-\mathrm{V}-1988$, J.J. Moldes Ferro s.n. MA 466035. SalamanCa: La Bastida, $40^{\circ} 35^{\prime} \mathrm{N}$, 6³'W, 7-VI-1971, F.J. Fernández Díez s.n., SALA 5605. Linares de Riofrío, $40^{\circ} 35^{\prime} \mathrm{N}, 5^{\circ} 55^{\circ} \mathrm{W}, 13$-VI-1969, Bellot \& Ron s.n., MA 634052. Masueco, $41^{\circ} 12^{\prime} \mathrm{N}$, 6 35'W, 16-V-1979, F. Amich s.n., SALA 16568. Montemayor del Río, $40^{\circ} 21^{\prime} \mathrm{N}, 5^{\circ} 53^{\prime}$ W, 17-V-1983, E. Rico \& A. Guillén s.n., MA 317754. Santibáñez de la Sierra, 40³0'N, $5^{\circ} 55^{\prime}$ 'W, 23-V-1971, F.J. Fernández Diez s.n., SALA 5325. Valle del río Alagón, $40^{\circ} 35^{\prime} \mathrm{N}, 5^{\circ} 50^{\prime} \mathrm{W}, 15-$ V-1971, Casaseca y cols. s.n., LEB 7409. Villarino de los Aires, 41 ${ }^{\circ} 16^{\prime} \mathrm{N}, 6^{\circ} 28^{\prime} \mathrm{W}, 27-\mathrm{IV}-1977$, J. Sánchez s.n., SALA 17918. SEvilla: Algámitas, Sierra del Tablón, $37^{\circ} 1^{\prime} \mathrm{N}, 5^{\circ} 9^{\prime} \mathrm{W}, 14-\mathrm{IV}-1978$, E. Ruiz de Clavijo \& B. Cabezudo s.n., SEV 32142. Constantina, arroyo del Huertano, Valcaliente, $37^{\circ} 52^{\prime} \mathrm{N}, 5^{\circ} 37^{\prime} \mathrm{W}, 5-\mathrm{V}-1984$, I. Lopez \& al. s.n., SEV 107082. Coripe, El Jerre, $36^{\circ} 58^{\prime} \mathrm{N}, 5^{\circ} 26^{\prime} \mathrm{W}$, 1-V-1933, C. Vicioso s.n., MA 24462. SorIA: Pedrajas, pr. Vellejo del Rey, $41^{\circ} 50^{\prime} \mathrm{N}, 2^{\circ} 35^{\prime} \mathrm{W}, 10-\mathrm{V}-1997$, Montamarta s.n., VAL 104768. Puerto del Madero, $41^{\circ} 49^{\prime} \mathrm{N}$, $2^{\circ} 7^{\prime}$ W, 9-V-1961, A. Segura Zubizarreta s.n., MA 359512. San Felices, $41^{\circ} 56^{\circ} \mathrm{N}, 2^{\circ} 2^{\prime}$ W, 8-V-1964, A. Segura Zubizarreta s.n., MA 359579. Valdeavellano de 
Tera, 4157'N, $2^{\circ} 35^{\prime}$ W, 7-VI-1968, A. Segura Zubizarreta s.n., MA 359522. TARRAGoNA: Arnes, Terra Alta, cap al Toll del Vidre i el Mas de'n Pau, 40 $54^{\circ} \mathrm{N}, 0^{\circ} 16^{\prime} \mathrm{E}$, 8-V-1983, J. Molero \& A. Rovira s.n., BCF 70763. Coll de la Teixeta, cap a Porrera, $41^{\circ} 9^{\prime} \mathrm{N}, 0^{\circ} 55^{\prime} \mathrm{E}, 2-\mathrm{V}-1982$, J. Molero \& A. Rovira s.n., BCF 50588. Els Motllats, a $23 \mathrm{~km}$ de Reus, 31-V-1981, J.A. Devesa \& al. 1618/81, SEV 71232. Entre Puig de la Mussara y Puig Pelat, $41^{\circ} 15^{\prime} \mathrm{N}, 1^{\circ} 3^{\prime} \mathrm{E}, 6-\mathrm{VI}-1954$, A. Bolòs s.n., BC 128711. Fredes al monte Caro. barranco de Millers, $40^{\circ} 42^{\prime} \mathrm{N}$, $0^{\circ} 10^{\prime}$ E, 8-VI-1999, C. Navarro \& al. CN2440, MA 626745. Muntanyes de Tivissa, $41^{\circ} 3^{\prime} \mathrm{N}, 0^{\circ} 44^{\prime} \mathrm{E}$. 28-IV1974, R. Folch s.n., BC 627421. Puertos de Beceite, El Retaule, $40^{\circ} 45^{\prime} \mathrm{N}, 0^{\circ} 18^{\prime} \mathrm{E}, 8-\mathrm{VI}-1986$, M.B. Crespo \& al. s.n., VAL 118168 B. Tortosa, $40^{\circ} 48^{\prime} \mathrm{N}, 0^{\circ} 31^{\prime} \mathrm{E}, 24-\mathrm{V}$ 1974, J. Fernández Casas s.n., MA 420679. TeRUEL: Albarracín, barranco del Cabrerizo, $40^{\circ} 28^{\prime} \mathrm{N}, 1^{\circ} 30^{\prime} \mathrm{W}, 14-$ V-1999, R. Giménez Martínez s.n., VAL 108233. Armillas, $40^{\circ} 53^{\prime} \mathrm{N}, 0^{\circ} 53^{\prime} \mathrm{W}, 16-\mathrm{V}-1990$, P. Montserrat s.n., JACA 99090 . Beceite, El Parrizal, $40^{\circ} 50^{\prime} \mathrm{N}, 0^{\circ} 11^{\prime} \mathrm{E}, 25-$ V-1991, M. Rubio s.n., VAL 75874. Calaceite, valle del Algás hacia Caseras, $41^{\circ} 1^{\prime} \mathrm{N}, 0^{\circ} 12^{\prime} \mathrm{E}, 27-\mathrm{IV}-1996$, G. Mateo \& al. 11291GM, VAL 97168. Formiche Alto, $40^{\circ} 19^{\prime} \mathrm{N}, 0^{\circ} 54^{\prime} \mathrm{W}, 17-\mathrm{V}-1992$, G. Mateo 6233, VAL 78051. Maicas, barranco Lavaderos, Puente Lipondo, $40^{\circ} 58^{\prime} \mathrm{N}, 0^{\circ} 53$ 'W, 7-V-1994, G. Mateo \& al. 8633GM, VAL 83748. Mora de Rubielos, $40^{\circ} 15^{\circ} \mathrm{N}, 0^{\circ} 45^{\prime} \mathrm{W}, 15-\mathrm{V}$ 1990, P. Montserrat s.n., JACA 84790. San Agustín, La Hoz, $40^{\circ} 3^{\prime} \mathrm{N}, 0^{\circ} 41^{\prime} \mathrm{W}, 11-\mathrm{V}-2000$, J. Riera \& F. Marco JRV3920, VAL 118101. Sierra de Albarracín, Valdecabriel, $40^{\circ} 18^{\prime} \mathrm{N}, 1^{\circ} 36^{\prime} \mathrm{W}$, Blanca \& Catalán s.n., MA 24446 B. TOLEDO: Villatobas, $39^{\circ} 54^{\prime} \mathrm{N}, 3^{\circ} 20^{\prime} \mathrm{W}, 21-\mathrm{V}$ 1968, P. Montserrat s.n., JACA 132468. VALENCIA: Castielfabib a Tormón, $40^{\circ} 8^{\prime} \mathrm{N}, 1^{\circ} 18^{\prime} \mathrm{W}, 23-\mathrm{V}-1988, \mathrm{G} . \mathrm{Ma}$ teo 707, VAL 59290 . Pinet, $38^{\circ} 59^{\prime} \mathrm{N}, 0^{\circ} 20^{\prime} \mathrm{W}, \mathrm{V}-1980$, Mansanet \& Mateo s.n., VAL 118169. ZAMORA: San Pedro de las Herrerías, 41 ${ }^{\circ} 54^{\prime} \mathrm{N}, 6^{\circ} 23^{\prime} \mathrm{W}, 11-\mathrm{V}-1982$, F. Navarro \& C.J. Valle s.n., SALA 3551. ZARAGOZA: barranco de la Sierra, $41^{\circ} 30^{\prime} \mathrm{N}, 1^{\circ} 37^{\prime} \mathrm{W}, 24-\mathrm{V}-1989$, P. Muñoz s.n., MA 556809. Calatayud, $41^{\circ} 21^{\prime}$ N, $1^{\circ} 38^{\prime} \mathrm{W}, 25-\mathrm{V}-1906$, C. Vicioso s.n., MA 24461. Cariñena, Puerto de Paniza, $41^{\circ} 20^{\prime} \mathrm{N}, 1^{\circ} 13^{\prime} \mathrm{W}, 25-\mathrm{V}-1980$, S. Castroviejo 1725SC, MA 295036. Cosuenda, Tobed, Sierra de Algairen, $41^{\circ} 22^{\prime} \mathrm{N}, 1^{\circ} 18^{\prime} \mathrm{W}, 10$-VI-1991, D. Gómez \& al. s.n., JACA 64891 . Gallocanta, $41^{\circ} 0^{\prime} \mathrm{N}$, $1^{\circ} 30^{\prime} \mathrm{W}, 27-\mathrm{V}-1981, D$. Gómez \& G. Montserrat s.n., JACA 574481 . Herrera de los Navarros, $41^{\circ} 12^{\prime} \mathrm{N}$, $1^{\circ} 5^{\prime} \mathrm{W}, 25-\mathrm{X}-1988, P$. Montserrat s.n., JACA 317588. Luesia, $42^{\circ} 22^{\prime} \mathrm{N}, 1^{\circ} 1^{\prime} \mathrm{W}, 23-\mathrm{V}-1975, P$. Montserrat \& L. Villar s.n., JACA 106275. Sierra de la Virgen, $41^{\circ} 32^{\prime} \mathrm{N}, 1^{\circ} 43^{\prime} \mathrm{W}, 10-\mathrm{VI}-1988$, P. Muñoz s.n., MA 556993. Sierra de Vicort, Calatayud, $41^{\circ} 22^{\prime} \mathrm{N}, 1^{\circ} 30^{\prime} \mathrm{W}$, 25-V-1906, C. Vicioso s.n., MA 24460.

MOR, Atlantis Majoris ditione Glaoua, Ait-Barka, $31^{\circ} 25^{\prime} \mathrm{N}, 7^{\circ} 17^{\prime} \mathrm{W}, \mathrm{IV}-1926, R$. Maire s.n., MPU. Haut Atlas centre, J. Tirardine, $31^{\circ} 9^{\prime} \mathrm{N}, 8^{\circ} 10^{\prime} \mathrm{W}, 26-\mathrm{V}-1952$, CH. Sauvage s.n., MPU 9600 . Tánger, Cala grande, $35^{\circ} 47^{\prime} \mathrm{N}, 5^{\circ} 49^{\prime} \mathrm{W}$, Broussonet s.n., MA 24471. Siadar, $35^{\circ} 23^{\prime}$ N, $2^{\circ} 54^{\prime}$ 'E, 29-III-1930, Mas Guindal s.n., MA 434057.

POR, ALTO ALENTEjo: Serta de Sao Mamede, próximo do Picoto, $39^{\circ} 19^{\prime} \mathrm{N}, 7^{\circ} 19^{\prime} \mathrm{W}, 29-\mathrm{IV}-1957$, Malato
Beliz \& al. s.n., MA 287679. BeIRA ALTA: Castro Daire, $40^{\circ} 54^{\prime} \mathrm{N}, 7^{\circ} 56^{\prime} \mathrm{W}$, IV-1886, M. Ferreira s.n., COI. Guarda, Souto do Bispo, 40 ${ }^{\circ} 32^{\prime} \mathrm{N}, 7^{\circ} 17^{\prime} \mathrm{W}, 23-\mathrm{IV}-1952$, A. Fermandes \& al. s.n., MA 287678. BEIRA LITORAL: Alfafar a Bandeira, pr. Coimbra, $40^{\circ} 4^{\prime} \mathrm{N}, 8^{\circ} 26^{\prime} \mathrm{W}, 12$-IV$1951, A$. Fernandes \& al. 3584, COI. Conraria, $40^{\circ} 10^{\prime} \mathrm{N}$, $8^{\circ} 24^{\prime}$ W, 9-IV-1952, U. Beau 534, COI. Figueira da Foz, $40^{\circ} 9^{\prime} \mathrm{N}, 8^{\circ} 52^{\prime} \mathrm{W}, \mathrm{IV}-1885$, F. Loureiro s.n., COI. Lagarteira, $39^{\circ} 57^{\prime} \mathrm{N}, 8^{\circ} 24^{\prime} \mathrm{W}$, III-1883, COI. Sernache, $40^{\circ} 8^{\prime} \mathrm{N}, 8^{\circ} 28^{\prime} \mathrm{W}, 20-\mathrm{V}-1889$, A.F. Perez 53, COI. Serra da Boa Viagem, próximo a Quiaios, $40^{\circ} 13^{\prime} \mathrm{N}, 8^{\circ} 51^{\prime} \mathrm{W}, 8$ IV-1975, A. Rozeira \& al. s.n., MA 293908. Tavarede, $40^{\circ} 10^{\prime} \mathrm{N}, 8^{\circ} 51^{\prime} \mathrm{W}, 16-\mathrm{III}-1957$, A. Fernandes \& al. 6151, COI. Douro Litoral: Casais do Douro, $41^{\circ} 10^{\prime} \mathrm{N}$, 7³2'W, 12-IV-1942, G. Pedro s.n., MA 412244. EsTREMADURA: Bombarral, $39^{\circ} 16^{\prime} \mathrm{N}, 9^{\circ} 9^{\prime} \mathrm{W}, 28-\mathrm{III}-1946$, B. Rainha 931, LISE 17484. Caldas de Rainha, $39^{\circ} 24^{\prime} \mathrm{N}$, $9^{\circ} 8^{\prime}$ W, III-1881, W.P. Lima s.n., COI. Cascaes, $38^{\circ} 42$ ' N, $9^{\circ} 25^{\prime} \mathrm{W}, \mathrm{II}-1950, U$. Beau 198. COI. Cintra, $38^{\circ} 48^{\prime} \mathrm{N}$, $9^{\circ} 23^{\prime}$ W, 28-IV-1944, B. Rainha s.n., MA 24470. Entre Cercal e Montejunto, $39^{\circ} 11^{\prime} \mathrm{N}, 9^{\circ} 3^{\circ} \mathrm{W}, 1-\mathrm{IV}-1963$, J. Paiva \& al. 8744, COI. Entre Porto de Mós e Mira d'Aire, 39'32'N. $8^{\circ} 43^{\prime} \mathrm{W}, 22-I V-1966$, A. Fermandes \& al. 9471 , COI. Lisboa, caminho novo, $38^{\circ} 43^{\prime} \mathrm{N}, 9^{\circ} 8^{\prime} \mathrm{W}$, 3-III-1962, U. Beau s.n., COI. Matinha de Queluz. $38^{\circ} 45^{\prime} \mathrm{N}, 9^{\circ} 15^{\prime} \mathrm{W}$, I-IV-1976, J. Cardoso s.n., BC 628745. Palmela. Serra de Sao Luis, $38^{\circ} 34^{\prime} \mathrm{N}, 8^{\circ} 54^{\prime} \mathrm{W}$, 23-III-1979, A.A. Ramos \& al. 263, LISE 89429. Setubal, Castelo de Sao Filippe, $38^{\circ} 32^{\prime}$ N. $8^{\circ} 54^{\prime}$ W, 1901, A. Luisier s.n., COI. Sierra de Sintra, Castello da Pena, $38^{\circ} 48^{\prime} \mathrm{N}, 9^{\circ} 23^{\prime} \mathrm{W}$, IV-1903, M. Rodríguez Lopez-Neyra s.n. MA 345611 . Tapada de Mafra, $38^{\circ} 56^{\prime} \mathrm{N}, 9^{\circ} 20^{\circ} \mathrm{W}$, IV-1885, $\boldsymbol{J}$. Guimaraes 765 , COI. Torres Vedras, $39^{\circ} 6^{\prime} \mathrm{N}, 9^{\circ} 16^{\prime} \mathrm{W}, 22-\Pi \mathrm{I}-1950$, A.R. Silva \& M. Silva s.n., MA 287535. Serra da Arrábida, $38^{\circ} 30^{\circ} \mathrm{N}, 9^{\circ} 0^{\prime} \mathrm{W}$, III1884, J. Daveau 1053, COI. MINHO: Chequeira, 23-IV1978, P. Cubas \& al. 53MG, MA 392973. Serra do Gerês, $41^{\circ} 50^{\prime} \mathrm{N}, 8^{\circ} 5^{\prime} \mathrm{W}, 15-\mathrm{VII}-1958$, Malato Beliz \& al. s.m. MA 192441. Serra do Gerês, Fonte Fria, $41^{\circ} 52^{\prime} \mathrm{N}$, $7^{\circ} 58^{\prime}$ 'W, 13-VII-1958, Malato Beliz \& al. s.n., MA 287680. Ribatejo: Tomar, pr. Pegoes, $39^{\circ} 36^{\prime} \mathrm{N}, 8^{\circ} 25^{\prime} \mathrm{W}$, 26-III1969. J. Martins 158. LISE 79081. Vale dos Lobos, Santarem, $39^{\circ} 14^{\prime} \mathrm{N}, 8^{\circ} 41^{\prime} \mathrm{W}, 19-\mathrm{II}-1966$, A.R. Moura 303, COI. Tras-os-Montes: Bragança, $41^{\circ} 49^{\prime} \mathrm{N}$, $6^{\circ} 45^{\prime} \mathrm{W}, 18-\mathrm{V}-1941$, A. Carneiro 160 , COI. Cova da Lua, intre Formos de Vilarinho et Alto do Mosqueiro, $41^{\circ} 53^{\prime} \mathrm{N}, 6^{\circ} 49^{\prime} \mathrm{W}, 19-\mathrm{VI}-1968$, Pinto da Silva \& A.N. Teles 8154 , LISE 66955. Pinhao, salida hacia Foz, $41^{\circ} 14^{\prime} \mathrm{N}$, $7^{\circ} 31$ 'W, 5-IV-2001, J.J. Aldasoro 2434, MA. Vimieiro, entre Bragança y Mirandela, $41^{\circ} 49^{\prime} \mathrm{N}, 6^{\circ} 45^{\prime} \mathrm{W}, 27$-IV1962, J. Paiva \& al. 8475, COI. Vimioso, entre Paradinha y Pinelo, $41^{\circ} 35^{\prime}$ N, $6^{\circ} 32^{\prime}$ W, 30-V-1908, J.M. Miranda 1089 , LISE 85635.

\section{Cephalanthera damasonium (Mill.)} Druce in Ann. Scott. Nat. Hist. 1906: 225 (1906)

Serapias damasonium Mill., Gard. Dict. ed. 8, n. ${ }^{\circ} 2$ (1768) basión.

Serapias lancifolia Murray, Syst. Veg. ed. 14: 815 (1784. Cephalanthera lancifolia 
(Murray) Tod., Orchid. Sicul.: 123 (1842. Epipactis lancifolia (Murray) All., Auct. Fl. Pedem.: 32 (1789)

Epipactis ochroleuca Baumg., Enum. Stirp. Transsilv. 3: 174 (1817). Cephalanthera ochroleuca (Baumg.) Rchb., Fl. Germ. Excurs. 1(1): $140^{20}(1831-32)$

Serapias pallens Jundz., Fl. Lith.: 268 (1791) [n.v.]

Epipactis alba Crantz, Stirp. Austr. Fasc. 2: 460 (1769). Cephalanthera alba (Crantz) Simonk., Enum. Fl. Transsilv.: 504 (1886) Serapias latifolia Mill., Gard. Dict. ed. 8, n. $^{\circ} 4$ (1768), nom. illeg. non Huds. (1762). Cephalanthera latifolia (Mill.) Janch. in Mitt. Naturwiss. Vereins. Univ. Wien 5(9): 111 (1907)

\section{Cephalantera grandiflora auct.}

Ind. loc.: "The second sort grows naturally in Stoken Church woods in Oxfordshire, and in several parts of Westmoreland and Lancashire" [neótipo, aquí designado, Inglaterra, Oxfordshire, Bledlow, near Thame, 29 Mayo 1960, Sims s.n. (BM, foto!)]

Ic.: Ross-Craig, Draw. Brit. Pl. 28, pl. 45 (1971)

Rizoma corto, horizontal, con abundantes raíces fibrosas, fasciculadas. Tallos (13)16$60 \mathrm{~cm}$, relativamente gruesos, estriados, macizos, verdes, casi glabros. Hojas (1)2-4(5) -más 1-3 reducidas a las vainas en la base-, de 4-9 × 1,4-3,8 cm, espaciadas, de ovales a lanceoladas -relación anchura/longitud $=$ 0,24-0,5-, agudas, margen entero, glabras, más largas y estrechas cerca de la inflorescencia. Inflorescencia (4)6-17(21) cm, (2)5-13 flores sésiles, \pm glabra; bráctea basal 4-9,5 $\times$ 1,3-2,6 cm, ovada -relación anchura/longitud $=0,16-0,43-$, foliácea, aguda, las restantes $1,7-9 \mathrm{~cm}$ de largo, más largas que el ovario -relación longitud tercera bráctea/longitud ovario $=1,3-2,5(4,2)-$, foliáceas, ovadas, agudas, glabras. Flores (2)2,8-4,5 cm de longitud. Sépalos $1,5-2,2 \times 0,4-0,75 \mathrm{~cm}$, oblongo-lanceolados, obtusos, casi glabros -con pelos glandulíferos dispersos en la cara externa-, blanco-crema. Pétalos laterales 1,4-1,8 $\times$ $0,45-0,5 \mathrm{~cm}$, algo menores que los sépalos, elípticos, obtusos, glabros, blanco-crema; la- belo 1,2-1,4 cm, menor que los sépalos, glabro; hipoquilo 0,5-0,6 × 1,2 cm, sacciforme en la base, con dos lóbulos laterales obtusos, que encierran totalmente el ginostemo, blanquecino; epiquilo 0,7-0,8 × $1 \mathrm{~cm}$-más ancho que largo-, reniforme, obtuso, blanquecino, con una mancha amarilla en la porción distal, con 3-5 crestas longitudinales de color amarillo anaranjado. Ginostemo 1-1,2 cm, cilíndrico, erecto, glabro. Antera c. $0,3 \mathrm{~mm}$, oblonga, cuculada; masas polínicas blanco-amarillentas. Ovario 1-2,3(3) $\mathrm{cm}$ de longitud, con pelos glandulíferos dispersos de $0,1-0,2 \mathrm{~mm}$. Fruto erecto, oblongo, con 6 costillas. Semillas $0,6-$ $1,1 \times 0,15-0,22 \mathrm{~mm}$.

Números cromosomáticos: $2 n=36$ (BIANCO \& al., 1987; MieIETA, 1986; RUIZ, 1995).

Hábitat: Bosques caducifolios, encinares, quejigares, sabinares y pinares; generalmente en suelos calizos, en lugares húmedos y umbrosos; (50)400-1700 m.

\section{Floración: IV-VIII(IX).}

Área de distribución: Casi toda Europa, SW de Asia, Cáucaso, Anatolia, Persia y NW de África. Mitad oriental de la Península -con una localidad extrema en Zamora- y Baleares.

Especie muy bien representada en el cuadrante NE de la Península, que se extiende hacia el W por el valle del Duero, y que se encuentra ocasionalmente en las montañas del SE. En las Islas Baleares solo se conoce de Mallorca, mientras que en el $\mathrm{N}$ de África su presencia consta únicamente en Argelia (MAIRE, 1959) (fig. 8).

No hemos podido estudiar material de la provincia de Albacete (cf. LÓPEZ VÉLEZ, 1984: 276).

El límite oriental parece situarse en Persia (RENZ, 1978: 26), aunque ha sido citada también en China (ZHU GUANGHUA, 1999: 80).

Según nos dice S. Cafferty (in litt.), en el herbario del Natural History Museum (BM) no se conserva ningún ejemplar relevante para la lectotipificación de la Serapias damasonium. El único ejemplar del propio Miller procede del Chelsea Physic Garden y no de alguna de las localidades señaladas en el protólogo. Por ello nos decidimos a proponer como neótipo un 
ejemplar procedente de Oxford, una de las zonas citadas por MILLER (1768) (fig. 9).

\section{Material estudiado}

ALG, Aurès, Sgag, $35^{\circ} 30^{\prime} \mathrm{N}, 6^{\circ} 10^{\prime} \mathrm{E}, 2$-VII-1920, R. Maire s.n., MPU.

SPA, ÁlAVA: Bernedo, 42³7'N, $2^{\circ} 30^{\prime} \mathrm{W}, \mathrm{VI}-1931$, M. Losa s.n., BCF 3766. Kanpezu, Santa Cruz de Campezo, $42^{\circ} 40^{\prime} \mathrm{N}, 2^{\circ} 2 \mathrm{l}^{\prime} \mathrm{W}, 2$-VI-1981, Puy Isaba s.n., ARAN 48643. Pipaon, $42^{\circ} 37^{\prime} \mathrm{N}, 2^{\circ} 39^{\prime} \mathrm{W}, \mathrm{VI}-1933$, M. Losa s.n., BCF 3767. Trespuentes, $42^{\circ} 51^{\prime} \mathrm{N}, 2^{\circ} 47$ ' W, 10-VI-1983, P.M. Uribe-Echebarria s.n., MA 523380. ALICANTE: Barranco de Alcolecha, 38 $41^{\prime} \mathrm{N}, 0^{\circ} 20^{\prime} \mathrm{W}, 3-\mathrm{VIII}-1976$, A. Rigual s.n., MA 373100 . Benifallim, Serra del Rentonar, $38^{\circ} 40^{\prime} \mathrm{N}, 0^{\circ} 24^{\prime} \mathrm{W}, 4-\mathrm{VI}-1989$, L. Serra s.n., VAL 118165. ALMERIA: Sierra María, $37^{\circ} 40^{\prime} \mathrm{N}, 2^{\circ} 13^{\prime} \mathrm{W}, 13-$ VI-1960, S. Rivas Goday s.n., MAF 82941. BALEARES: Mallorca, Escorca al Gorg Blau, $39^{\circ} 49^{\prime} \mathrm{N}, 2^{\circ} 51^{\prime} \mathrm{E}, 12-\mathrm{V}-$ 1947, Palau Ferrer s.n., MA 345538. Lluch, 39 $49^{\circ}$ N, $2^{\circ} 53$ 'E, 4-V-1947, Palau Ferrer s.n., MA 24430. BARCELONA: Montserrat, $41^{\circ} 35^{\prime}$ N, $1^{\circ} 46^{\prime} \mathrm{E}, 12$-VII-1903, Marcet s.n., MA 24427 . Montseny, $41^{\circ} 46^{\prime} \mathrm{N}, 2^{\circ} 24^{\prime} \mathrm{E}$, VII1917, F. Sennen s.n., BC 837523. Osona, Tona, Turó del Castell, 41 ${ }^{\circ} 51^{\prime} \mathrm{N}, 2^{\circ} 14^{\prime} \mathrm{E}, 8-\mathrm{VI}-1952$, A. Bolòs \& O. Bolòs s.n., BC 118780. Sant Cugat, $41^{\circ} 28^{\prime} \mathrm{N}, 2^{\circ} 5^{\prime} \mathrm{E}$, IV-1926, F. Sennen s.n., BC 837522. Sant Llorens del
Munt, $41^{\circ} 38^{\prime} \mathrm{N}, 2^{\circ} 1^{\prime} \mathrm{E}$, , M. Losa s.n., BCF 51221 . BuRGos: Bañuelos del Rudrón, $42^{\circ} 41^{\prime} \mathrm{N}, 3^{\circ} 51^{\prime} \mathrm{W}, 28-\mathrm{VI}$ 1988, P. Galán Cela \& G. López 3I7IPG, MA 440630. Bujedo, valle de la $\mathrm{Hoz}, 42^{\circ} 39^{\prime} \mathrm{N}, 3^{\circ} 10^{\prime} \mathrm{W}$, M. Losa s.n., BCF 3765 . Burgos, $42^{\circ} 21^{\circ} \mathrm{N}, 3^{\circ} 42^{\prime} \mathrm{W}, \mathrm{VI}-1984$, C. Aedo s.n. MA 598359. Camino de Fuenteoco a El Gricio, $41^{\circ} 45^{\prime} \mathrm{N}, 4^{\circ} 6^{\prime} \mathrm{W}, 5-\mathrm{VI}-1993$, J.L. Fernández Alonso \& R. Castillo 10612, MA 525212. Fuente de la Salud, $42^{\circ} 21^{\prime} \mathrm{N}, 3^{\circ} 40^{\prime} \mathrm{W}, 21-\mathrm{VI}-1914$, P. Font Quer s.n., BC 60116. Parque de Fuentes Blancas, $42^{\circ} 20^{\prime} \mathrm{N}, 3^{\circ} 40^{\prime} \mathrm{W}$, 22-VI-1985, L. Marín s.n.. MA 294707. Quintanar de la Sierra, 41 ${ }^{\circ} 59^{\prime}$ N, 32' W, 13-VII-1972, A. Segura Zubizarreta s.n., MA 359525. Quintanilla del Coco, $41^{\circ} 59^{\prime} \mathrm{N}$, $3^{\circ} 31^{\prime} \mathrm{W}, 1-\mathrm{VI}-1986$. L. Marin s.n., MA 311165 . Santo Domingo de Silos, Oña, $41^{\circ} 58^{\prime} \mathrm{N}, 3^{\circ} 25^{\prime} \mathrm{W}$, Losa? s.m., BCF 3770 . Valle de Valdivielso, cerca de Valhermosa, $42^{\circ} 49^{\prime} \mathrm{N}, 3^{\circ} 31$ ' W, 30-V-1986, L. Marín s.n., MA 311166 . Villaescusa de Roa, $41^{\circ} 44^{\prime} \mathrm{N}, 4^{\circ} 1^{\prime} \mathrm{W}, 15-\mathrm{V}$ 1983. J.L. Fernández Alonso 1394, MA 517276. CANTABRIA: Bernedo, $42^{\circ} 37^{\prime} \mathrm{N}, 2^{\circ} 30^{\prime} \mathrm{W}, 9$-VI-1986, A, Alejandre s.n., MA 365882. San Martín de Elines, pr. Villaescusa de Ebro, 42 $50^{\prime}$ N, $3^{\circ} 51^{\prime}$ W, 28-V-1991, M. Lainz \& Ó. Sánchez Pedraja s.n., herb. Sánchez Pedraja 1686. Castellón: Alt Millars, Fuente la Reina, $40^{\circ} 4^{\prime} \mathrm{N}$, $0^{\circ} 377^{\prime} \mathrm{W}, 3-\mathrm{VI}-1989, \mathrm{~J}$. Riera $934 J R V$, VAL 21539. Alt Palància, Pina de Montalgrao, El Collado, $40^{\circ} 1$ 'N, $0^{\circ} 39^{\prime} \mathrm{W}, 27-\mathrm{V}-1989$, J. Riera 617JRV, VAL 21536. Ares del Maestre, alrededores del Mas de les Pallises,

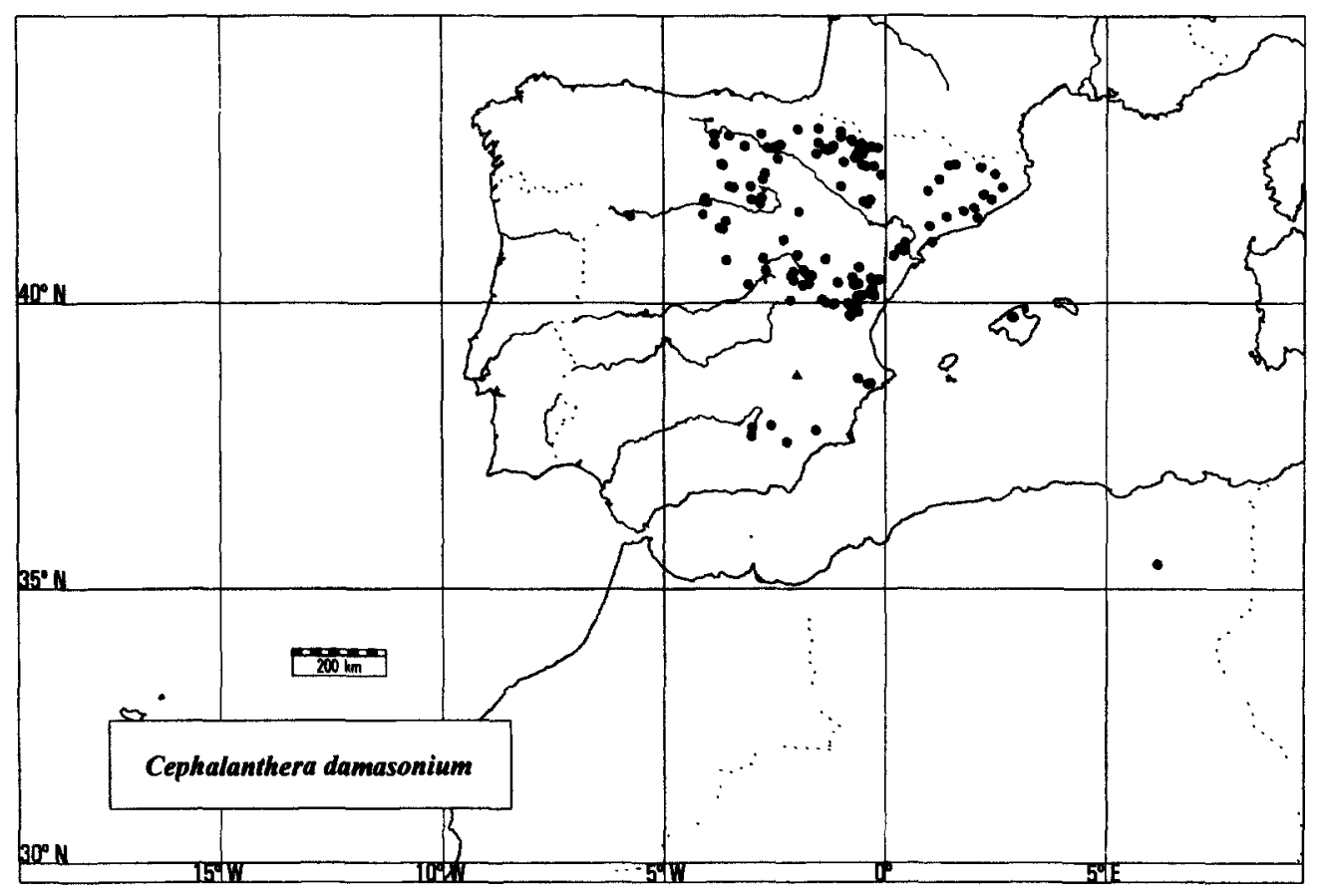

Fig. 8.-Distribución de Cephalanthera damasonium en la Península Ibérica y N de África (•, material estudiado; $\Delta$, citas bibliograficas). 


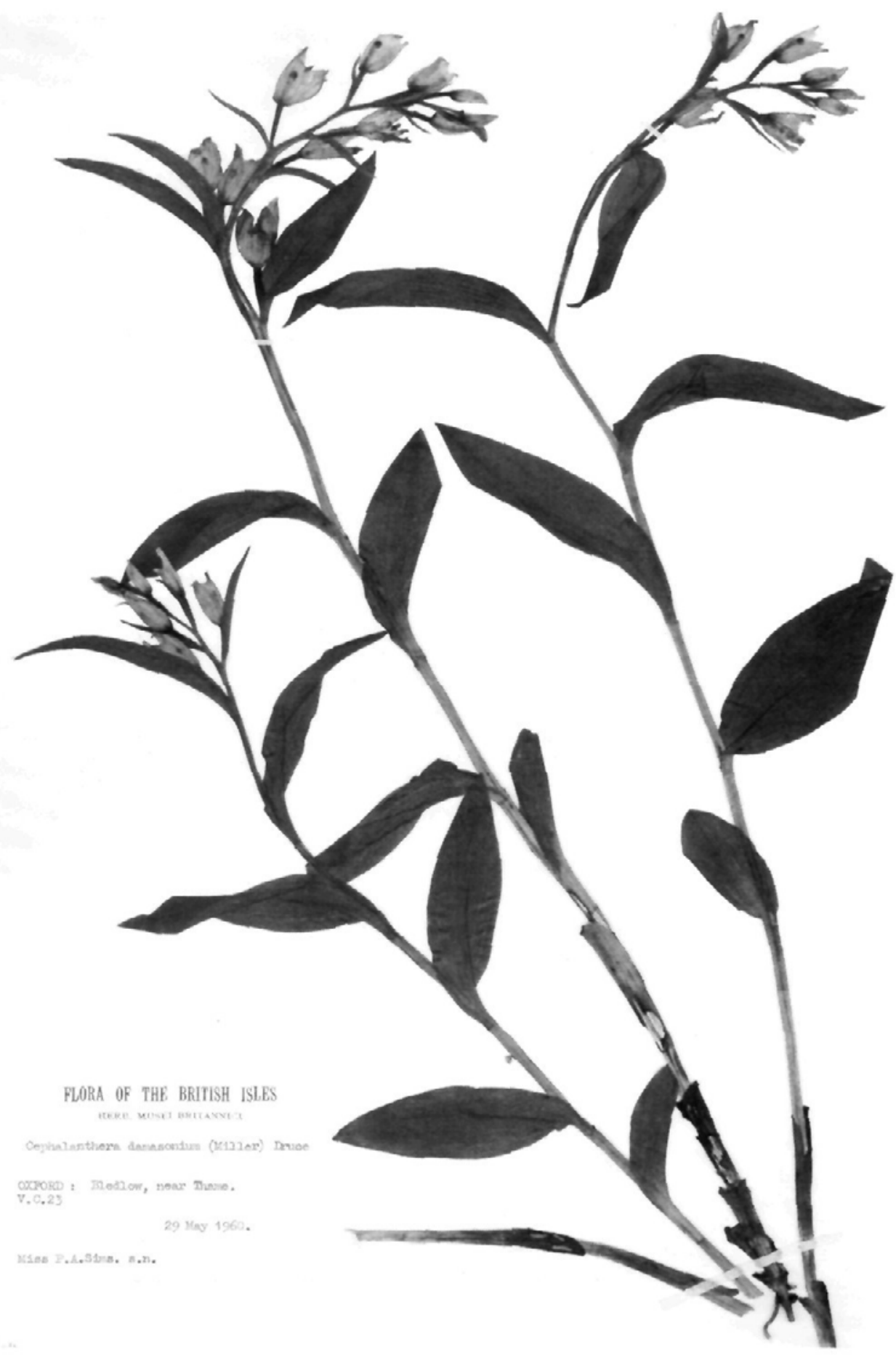

Fig. 9.-Neótipo de Serapias damasonium Mill. 
$40^{\circ} 27^{\prime} \mathrm{N}, 0^{\circ} 8^{\prime} \mathrm{W}, 18-\mathrm{V}-1991$, C. Fabregat \& S. López 2017CF, VAL 89854. Barranco de Horta, pr. San Miguel de la Puebla, $40^{\circ} 28^{\prime} \mathrm{N}, 0^{\circ} 19^{\prime} \mathrm{W}, 1-\mathrm{VII}-1987$, G. Mateo \& R. Figuerola s.n., VAL 55952. Bejís, río Palancia, $39^{\circ} 54^{\prime} \mathrm{N}, 0^{\circ} 42^{\prime} \mathrm{W}, 27-\mathrm{V}-2000$, J. Andrés \& al. 68JNT, VAL 42942. Cortes de Arenoso, Pico de Cruces, $40^{\circ} 11^{\prime} \mathrm{N}, 0^{\circ} 32^{\prime} \mathrm{W}, 24-\mathrm{Vl}-1992$, G. Mateo \& al. 6401, VAL 78297, El Toro, entre Peña de las Majadas y la Peña del Clavo, 39 $59^{\prime} \mathrm{N}, 0^{\circ} 45^{\prime} \mathrm{W}, 27-\mathrm{V}-2000$, J. Andrés \& al. 71JNT, VAL 43030. L'Alcalatén-El Xaparrar, 40 11 'N, $0^{\circ} 14^{\prime} \mathrm{W}, 20-\mathrm{VI}-1980, \mathrm{~J}$. Vigo \& al. s.n., BC 640800. Penagolosa, $40^{\circ} 13^{\prime} \mathrm{N}, 0^{\circ} 21^{\prime} \mathrm{W}$, A. Cavanilles s.n., MA 24420. Villafranca del Cid, barranco de las Tejerías, $40^{\circ} 25^{\prime} \mathrm{N}, 0^{\circ} 15^{\prime} \mathrm{W}, 2$-VI-1990, C. Fabregat s.n., VAL 68837. Vistabella del Maestrazgo, $40^{\circ} 18^{\prime} \mathrm{N}, 0^{\circ} 17^{\prime} \mathrm{W}$, A. Cavanilles s.n., MA 24446 B. Viver, cuestas del Ragudo, 3955'N, 0³6'W, 15-VII-1984, A. Aguilella s.n., VAL 5991. CuenCA: Balneario de Solán de Cabras, $40^{\circ} 30^{\prime} \mathrm{N}, 2^{\circ} 7^{\prime} \mathrm{W}, 5-\mathrm{VI}-1975$, G. López \& al. 1209GF, MA 393027. Beteta, Hoz de Beteta, 4034'N, $2^{\circ} 4^{\prime} \mathrm{W}, 9-$ VI-1989, Gamarra \& al. s.n., MA 472032. Poyatos, 40²6'N, 24'W, 15-VI-1982, E. Rico \& J. Sánchez s.n., SALA 32100. Sierra del Tremedal, Hoz del Tajo, $40^{\circ} 31^{\prime} \mathrm{N}, 1^{\circ} 39^{\prime} \mathrm{W}, 9-\mathrm{VII}-1979$, G. López 1557GF, MA 393057. Tragacete, $40^{\circ} 21^{\prime} \mathrm{N}, 1^{\circ} 51^{\prime} \mathrm{W}, 19-\mathrm{VII}-1975$, L. Villar s.n., JACA 349775. Valle del Júcar, $40^{\circ} 6^{\prime} \mathrm{N}$, 2'8'W, 4-V-1997, G. Mateo \& Hernández Viadel s.n, VAL 102004. GERONA: Olot, 42 $11^{\prime} \mathrm{N}, 2^{\circ} 29^{\prime} \mathrm{E}$, IV-1872, S. Vayreda s.n., BC 60119 . Sant Juliá del Llor, $41^{\circ} 58^{\prime} \mathrm{N}$, $2^{\circ} 39^{\prime}$ E, 16-V-1920, s.n., BC 60120. Vall de Ribes, Srta. Barricó, 42 $18^{\prime} \mathrm{N}, 2^{\circ} 10^{\prime} \mathrm{E}, 11$-VI-1970, J. Vigo s.n. BC 605785. GRanada: Macizo de la Sagra, $37^{\circ} 57^{\prime} \mathrm{N}$, 2³4'W, 16-VI-1960, S. Rivas Goday \& al. s.n. MAF 69884. Guadalajara: Barriopedro, $40^{\circ} 48^{\prime} \mathrm{N}, 2^{\circ} 45^{\prime} \mathrm{W}$, 23-VI-1970, Bellot \& al. s.n., MA 95269. Chequilla, 40³6'N. 1'50'W, 21-VI-1995, M.A. Carrasco \& al. 682FCL, MA 558807. Chillarón del Rey, 40³6'N, $2^{\circ} 41^{\prime}$ W, 21-VI-1970, Bellot \& al. s.n., MA 95270. Corduente, barranco de la Hoz, 40 $51^{\prime} \mathrm{N}, 1^{\circ} 59^{\prime} \mathrm{W}, 23-\mathrm{V}$ 1998, Pisco s.n, VAL 107617. Fuentenovilla, $40^{\circ} 22^{\prime} \mathrm{N}$, 35'W, 6-VI-1970, Bellot \& al. s.n., MA 95271. HuEsCA: Aratorés, subida a Las Blancas, $42^{\circ} 39^{\prime} \mathrm{N}, 0^{\circ} 34^{\prime} \mathrm{W}, 30$ VI-1991, J. Amigo s.n., SANT 21117. Arguis, $42^{\circ} 19^{\prime} \mathrm{N}$, $0^{\circ} 26^{\prime} \mathrm{W}, 2-\mathrm{IX}-1987$, L. Villar \& al. s.n., JACA 347787. Baraguás, Jaca, $42^{\circ} 35^{\prime} \mathrm{N}, 0^{\circ} 29^{\prime} \mathrm{W}, 16-\mathrm{VII}-1969$, P. Montserrat s.n., JACA 435969. Bentué de Rasal, monte Peiró, 42 $20^{\circ} \mathrm{N}, 0^{\circ} 31^{\prime} \mathrm{W}, 21-\mathrm{VI}-1974, L$. Villar s.n., JACA 297174. Bierge, Rodellar, $42^{\circ} 10^{\prime} \mathrm{N}, 0^{\circ} 5^{\prime} \mathrm{W}, 10-\mathrm{V}$ 1967, P. Montserrat s.n., JACA 277567. Biescas, $42^{\circ} 38^{\prime}$ N, $0^{\circ} 19^{\prime} \mathrm{W}, 24-\mathrm{VI}-1993$, G. Mateo \& $P$. Montserrat $8333 G M$, VAL 81584 . Ena, Ordaniso, $42^{\circ} 27^{\prime} \mathrm{N}$, $0^{\circ} 41^{\prime}$ W. 28-V-1973, P. Montserrat s.n., JACA 138473. Hecho, Agüerri, $42^{\circ} 44^{\prime} \mathrm{N}, 0^{\circ} 45^{\prime} \mathrm{W}, 25-\mathrm{VII}-1974, L$. Villar s.n., JACA 10067674 B. Jaca, 42 $34^{\circ}$ N, $0^{\circ} 33^{\prime} \mathrm{W}$, VI1943, A. Bolòs s.n., BC 115088. Lanaja, 41 $41^{\circ}$ 'N, $0^{\circ} 20^{\prime}$ W, 20-IX-1996, J.V. Ferrández s.n., JACA 383496. Linas de Broto, $42^{\circ} 37^{\prime} \mathrm{N}, 0^{\circ} 10^{\prime} \mathrm{W}, 26-$ VII-1991, P. Montserrat \& al. s.n., JACA 156291. Monegros, Sierra de Alcubierre, al W de San Capracio, 41 ${ }^{\circ} 44^{\prime} \mathrm{N}$, 0²9’W. 9-VI-1996, J. Molero \& L Sáez s.n., BCF 41715 A. Nocito, Guara, $42^{\circ} 19^{\prime} \mathrm{N}, 0^{\circ} 15^{\prime} \mathrm{W} .11-\mathrm{VII}-1974$, P. Montserrat s.n., JACA 371374 . Oroel. Jaca, $42^{\circ} 31^{\prime} \mathrm{N}$,
0³1'W, 24-VI-1970, P. Montserrat s.n., JACA 274170. San Juan de la Peña, 42 $31^{\prime}$ N, 0 $38^{\prime}$ W, 16-VI-1942, A. Bolòs s.n., BC 95973. Sierra de Lanaja, bco. del Bu-

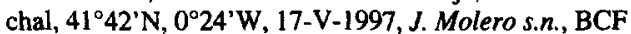
42442. Siresa, La Reclusa, Hecho, $42^{\circ} 45^{\prime} \mathrm{N}, 0^{\circ} 45^{\prime} \mathrm{W}, 28$ VI-1975, L. Villar s.n., JACA 10053275. Villanúa, $42^{\circ} 41^{\prime} \mathrm{N}, 0^{\circ} 32^{\prime} \mathrm{W}, 8$-VIII-1984, L. Villar s.n., JACA 123484. JAÉN: Cazorla, Cerro de La Laguna, $37^{\circ} 55^{\prime} \mathrm{N}$, 30'W, 22-VI-1975, González Rebollar \& al. s.n., MA 481208. Tíscar, $37^{\circ} 46^{\prime} \mathrm{N}, 3^{\circ} 1^{\prime} \mathrm{W}, 14-\mathrm{V}-1983$, M.A. Hernández s.n., MA 293895. LA RiOJA: Puente Madre, $42^{\circ} 26^{\prime} \mathrm{N}, 2^{\circ} 25^{\prime} \mathrm{W}, \mathrm{V}-1911$, I. Zubia s.n., MA 24425 . Rasillo de Cameros, $42^{\circ} 12^{\prime} \mathrm{N}, 2^{\circ} 42^{\prime} \mathrm{W}$, VI-1924, s.n., MA 24446 A. LÉrIDA: Alòs de Balaguer, $41^{\circ} 55^{\prime} \mathrm{N}, 0^{\circ} 58^{\prime} \mathrm{E}$, 27-V-1984, J. Pedrol s.n., MA 419417. Arséguel, $42^{\circ} 21^{\prime} \mathrm{N}, 1^{\circ} 36^{\prime} \mathrm{E}, 18-\mathrm{V}-1992$, P. Montserrat s.n., JACA 46492. Castellciutat, $42^{\circ} 20^{\prime} \mathrm{N}, 1^{\circ} 27^{\prime} \mathrm{E}, 14-\mathrm{V}-1972$, P. Montserrat \& L. Villar s.n., JACA 114272. Serra d'Aubenç, obaga del Mostalló, 42 ${ }^{\circ} 6^{\prime} \mathrm{N}, 1^{\circ} 14^{\prime} \mathrm{E}, 12-\mathrm{VI}-$ 1977, Molero \& Silvestre s.n., BCF 51226. MADRID; El Vellón, 4046'N, 335'W, 29-V-1985, F. Gómez Manzaneque s.n., MA 453088. MURCIA: Sierra de Espuña, subida al Morón de Espuña, 3752'N, $1^{\circ} 34^{\prime} W, 4-V-1979$, F. Garcia \& al. s.n., SEV 161647. NAvarRA: Aibar, Olatz, 42 $36^{\circ} \mathrm{N}, 1^{\circ} 21^{\prime} \mathrm{W}, 12-\mathrm{V}-1986$, I. Aizpuru \& Catalán s.n., ARAN 32502. Esteribar (Zubiri), AntxorizArromendi, 42 ${ }^{\circ} 56^{\prime} \mathrm{N}, 1^{\circ} 30^{\prime} \mathrm{W}, 12-\mathrm{VI}-1987$. I. Aizpuru \& Catalán s.n. ARAN 32500. Huarte-Araquil, San Donato, $42^{\circ} 55^{\prime} \mathrm{N}, 1^{\circ} 58^{\prime} \mathrm{W}, 27-\mathrm{VI}-1972$, P. Montserrat \& L Villar s.n., JACA 360872 . Javier, Peña, $42^{\circ} 36^{\circ} \mathrm{N}, 1^{\circ} 13^{\prime} \mathrm{W}$, 8-VI-1985, I. Aizpuru \& al. s.n., ARAN 32505. Lapoblación, El Castillo, $42^{\circ} 36^{\prime} \mathrm{N}, 2^{\circ} 27^{\prime} \mathrm{W}, 24-\mathrm{VI}-1985$, I. Aizpuru \& al. s.n., ARAN 32504. Leyre, 42 $39^{\prime} \mathrm{N}, 1^{\circ} 10^{\prime} \mathrm{W}$, 23-IX-1972, P. Montserrat \& L. Villar s.n., JACA 735572. Marañón, 42 38'N, 2²6'W, 12-VI-1981, Puy Isaba s.n., ARAN 48642 B. Monreal, Arbiain, $42^{\circ} 42^{\prime} \mathrm{N}$, 1³0'W, 18-V-1985, I. Aizpuru \& al. s.n., ARAN 32506. San Martín de Unx, 42 $31^{\prime}$ N, $1^{\circ} 33^{\prime}$ W, 5-VI-1986, I. Aizpuru \& Catalán s.n., ARAN 32503. Sangüesa, Sarda, $42^{\circ} 35^{\prime} \mathrm{N}, 1^{\circ} 17^{\prime} \mathrm{W}, 26-\mathrm{V}-1987,1$. Aizpuru \& Catalán s.n., ARAN 32501. Uztarroz-Iza Izu, 42 $53^{\prime} \mathrm{N}, 1^{\circ} 0^{\prime} \mathrm{W}, 8$-VII1971, L. Villar s.n., JACA 10128571. Valle del Urederra, Amezketa, $42^{\circ} 42^{\prime}$ N. $2^{\circ} 3^{\prime}$ W, 10-VI-1984, P. Garin s.n., ARAN 32507. Vidangoz, $42^{\circ} 48^{\prime} \mathrm{N}, 1^{\circ} 0^{\prime} \mathrm{W}, 11-\mathrm{VI}-1989$, J. Loidi \& al. s.n., MA 536867. PALENCIA: Castrillo de Don Juan, 41 ${ }^{\circ} 48^{\prime}$ 'N. 44'W, 16-VII-1982, J.L. Fernández Alonso 157JF, MA 294278. SEgovia: Cañón del Duratón, $41^{\circ} 17 '$ N, $3^{\circ} 41^{\prime} \mathrm{W}, 2-\mathrm{VI}-1983$, M. Luceño s.n., MA 393021 . Cedillo de la Torre, $41^{\circ} 25^{\prime} \mathrm{N}, 3^{\circ} 36^{\prime} \mathrm{W}, 27-\mathrm{VI}-$ 1985, A. Izuzquiza s.n., MA 506961. Sepúlveda, Hoz del Duratón, $41^{\circ} 18^{\prime} \mathrm{N}, 3^{\circ} 45^{\prime} \mathrm{W}, 21-\mathrm{V}-1981, C$. Cebolla \& al. s.n., MA 366307. Soria: Abejar, $41^{\circ} 48^{\prime} \mathrm{N}, 2^{\circ} 47^{\prime} \mathrm{W}, 20$ V-1961, A. Segura Zubizarreta s.n., MA 359523. Calatañazor, $41^{\circ} 42^{\prime} \mathrm{N}, 2^{\circ} 49^{\prime} \mathrm{W}, 4-\mathrm{VII}-1984, E$. Rico \& T. Romero s.n., MA 567154 . Herrera de Soria, $41^{\circ} 46^{\prime} \mathrm{N}$, $3^{\circ} 1$ 'W, 5-VI-1986, A. Buades s.n., MA 551704. Layna, hacia Maranchón, $41^{\circ} 6^{\prime} \mathrm{N}, 2^{\circ} 18^{\prime} \mathrm{W}, 30-\mathrm{V}-1992$, G. $M a-$ teo \& al. 6159. VAL 77948. Montenegro de Cameros, $42^{\circ} 6^{\prime} \mathrm{N}, 2^{\circ} 45^{\prime} \mathrm{W}, 26-\mathrm{VI}-1968$, A. Segura Zubizarreta s.n., MA 359583. TARRagona: Cambrils, $41^{\circ} 4^{\prime} \mathrm{N}, 1^{\circ} 3^{\prime} \mathrm{E}$, V-1918, F. Sennen s.n., BC 837521 B. Gandesa, Terra Alta, El Montalvo, Serra de Pandols, $41^{\circ} 3^{\prime} \mathrm{N}, 0^{\circ} 26^{\prime} \mathrm{E}$, 
29-V-1982, J. Molero \& A. Rovira s.n., BCF 70785. Horta de San Juan, Les Heres, $40^{\circ} 57^{\prime}$ N, $0^{\circ} 19^{\prime} E$, 27-VII1982, J. Molero \& A. Rovira s.n., BCF 70762. Pontils, $41^{\circ} 29^{\prime} \mathrm{N}, 1^{\circ} 23^{\prime} \mathrm{E}, 25-\mathrm{V}-1947$, E. Batalla \& F. Mansclans s.n., BC 103995. Ports de Paüls. Serra d'Alfara, $40^{\circ} 55^{\prime}$ N. $0^{\circ} 24 '$ 'E, 26-IV-1982, J. Molero \& A. Rovira s.n., BCF 70787. Muntanyes de Prades, Massís de Roquerola, $41^{\circ} 20^{\prime} \mathrm{N}, 1^{\circ} 0$ 'E, 28-V-1950, E. Batalla \& F. Mansclans s.n., BC 601374 . TERUEL: Alcalá de la Selva, $40^{\circ} 22^{\prime} \mathrm{N}$, $0^{\circ} 42^{\prime}$ W, 9-VII-1957, P. Montserrat \& al. s.n. JACA 155357 A. barranco entre Alcotas y Manzanera, $40^{\circ} 1^{\circ} \mathrm{N}$, $0^{\circ} 47$ 'W, 11-VI-1984, A. Aguilella s.n., VAL 5993. Beceite. Valle del río Ulldemó, $40^{\circ} 50^{\prime} \mathrm{N}, 0^{\circ}-11$ 'E, 5-VI1991, G. Mateo \& S. López 4290. VAL 73970. Fuentes de Rubielos, Torre Batán, $40^{\circ} 10^{\prime} \mathrm{N}, 0^{\circ} 37^{\prime} \mathrm{W}, 3-\mathrm{VI}-1995$, Marín Campos s.n., VAL 106307. Guadalaviar, Sierra de Albarracín, 40 ${ }^{\circ} 23^{\prime} \mathrm{N}, 1^{\circ} 43^{\prime} \mathrm{W}, \mathrm{V}-1908$, ¿Capicol? s.n., MA 433943. Manzanera, Fuente Roya, Los Paraísos, $40^{\circ} 3^{\prime} \mathrm{N}, 0^{\circ} 50^{\prime} \mathrm{W}, 7-\mathrm{VII}-1984$, A. Aguilella s.n., VAL 5990. Monreal del Campo, $40^{\circ} 47^{\prime} \mathrm{N}, 1^{\circ} 21^{\prime} \mathrm{W}$, Benedicto s.n., BC 60102. Pitarque, $40^{\circ} 39^{\prime} \mathrm{N}, 0^{\circ} 35^{\prime} \mathrm{W}, 10-\mathrm{VI}-1986$, J. Güemes s.n., VAL 4609 . Sollavientos, $40^{\circ} 29^{\prime} \mathrm{N}$, $0^{\circ} 44^{\prime} \mathrm{W}, 3-\mathrm{VII}-1957$, P. Montserrat \& al. s.n., JACA 138357A. Tortajada, vega del Alfambra, $40^{\circ} 24^{\prime} \mathrm{N}$, $1^{\circ} 4^{\prime} \mathrm{W}, 25-\mathrm{V}-1995$, G. Moreno Valdeolivas s.n., VAL 92919. Valdelinares, $40^{\circ} 23^{\prime} \mathrm{N}, 0^{\circ} 36^{\prime} \mathrm{W}, 18-\mathrm{VI}-1983$, A. Aguilella \& al. s.n., VAL 1171. VALENCIA: Andilla, Collado de la Salada, $39^{\circ} 50^{\prime} \mathrm{N}, 0^{\circ} 48^{\prime} \mathrm{W}, 26-\mathrm{V}-1982$, A. Aguilella s.n., VAL 6000 . Arroyo Cerezo, $40^{\circ} 7^{\prime} \mathrm{N}$, $1^{\circ} 25^{\prime} \mathrm{W}, 15-\mathrm{VI}-1988, G$. Mateo \& al. 222, VAL 58759. Bocairent, La Vall d'Albaida, $38^{\circ} 46^{\prime} \mathrm{N}, 0^{\circ} 37^{\prime} \mathrm{W}, 3-\mathrm{V}$ 1987, J.R. Nebot s.n., VAL 63584. El Cabezo de Arroyo Cerezo, $40^{\circ} 7{ }^{\prime} \mathrm{N}, 1^{\circ} 25^{\prime} \mathrm{W}, 15-\mathrm{VI}-1988$, G. Mateo \& al. 189, VAL 58001. Puebla de San Miguel, $40^{\circ} 3 \mathrm{~N}, 1^{\circ} 8^{\prime} \mathrm{W}$, 30-V-1986, G. Mateo s.n, VAL 118163 . Sesga, $40^{\circ} 2^{\prime}$ N, $1^{\circ} 11$ 'W, 22-V-1988, G. Mateo 882, VAL 59490. Vallanca, 40 4' N, 1'20'W, 4, G. Mateo \& R. Figuerola 40, VAL 118162. ValLadolid: Canalejas de Peñafiel, $41^{\circ} 32^{\prime} \mathrm{N}, 4^{\circ} 7^{\prime} \mathrm{W}, 15-\mathrm{VI}-1984$, T. Romero s.n., MA 567041. Encinas de Esgueva, $41^{\circ} 45^{\prime} \mathrm{N}, 4^{\circ} 6^{\prime} \mathrm{W}, 5$-VI1993, J.L. Fernández-Alonso 10595, MA 525228. ZAMORA: Zamora, $41^{\circ} 30^{\prime} \mathrm{N}, 5^{\circ} 45^{\prime} \mathrm{W}, 1940$, E. Sierra s.n., BC 93992. Zaragoza: Berdejo, $41^{\circ} 34^{\prime} \mathrm{N}, 1^{\circ} 57^{\prime} \mathrm{W}, 5$-VI1969, A. Segura Zubizarreta s.n., MA 359580. Biel, Paco de la Selva, $42^{\circ} 23^{\prime} \mathrm{N}, 0^{\circ} 56^{\prime} \mathrm{W}, 1$-VII-1987, $L$. Villar \& al. s.n., MA 515688. Castejón de Valdejasa, $41^{\circ} 59^{\prime} \mathrm{N}$, $1^{\circ} 0^{\prime} \mathrm{W}, 12-\mathrm{VI}-1973$, L Villar \& A. Lanaspa s.n., JACA 168073.

\section{AgRADECIMIENTOS}

Queremos agradecer a los conservadores de los herbarios consultados que amablemente nos enviaron el material disponible; a F. Muñoz Garmendia, por su ayuda con la nomenclatura, y a S. Cafferty, por su colaboración en los tipos. Una primera versión de este artículo fue corregida por J.J. Aldasoro y C. Navarro, quienes aportaron valiosas sugerencias; dos revisores anónimos contribuyeron también a mejorar el artículo con numerosas correccio- nes. L. Sáez revisó cuidadosamente las localidades de Cataluña y Baleares. Este trabajo ha sido financiado a través del proyecto de investigación Flora Iberica (PB-1996-0849).

\section{REFERENCIAS BIBLIOGRÁFICAS}

Aedo, C., M. ARguelles, J.M. GonzÁlez del Valle \& M. LAf́nZ (1990). Aportaciones al conocimiento de la flora de Asturias II. Collect. Bot. (Barcelona) 18: 99 116.

asegnolaza, C., D. Gómez, X. Lizaur, G. MontserRat, G. Morante, M.R. Salaverrfa, P.M. UribeECHEBARRIA \& J.A. AlEJANDRE (1985). Catálogo florístico de Álava, Vizcaya y Guipúzcoa. Vitoria.

Averyanov, L.V., N.A. MEdvedeva \& V.P. Serov (1985). Chromosome numbers in the representatives of the family Orchidaceae from the Caucasus. Bot. Zhum. SSSR. 70(7): 999-1000.

BaUmanN, H., S. KúNKele \& R. LoRenz (1989). Die nomenklatorischen Typen der von Linnaeus veröffentlichten Namen europäischer Orchideen. Mitt. Arbeitskreis Heimische Orchid. Baden-Württemberg 21: 355-700.

Bianco, P., P. Medagli, S. D'Emerico \& L. RugGiero (1987). Numeri cromosomici per la flora Italiana: 1139-1155. Inform. Bot. Ital. 19: 322-332.

Cameron, K.M., M.W. Chase, W.M. Whitten, P.J. KoRES, D.C. JARRELL, V.A. ALBERT, T. YUKAWA, H.G. HILlS \& D.H. GoldMaN (1999). A phylogenetic analysis of the Orchidaceae: evidence from rbcL nucleotide sequences. Amer. J. Bot. 86: 208-224.

CAPINERI, R. \& W. Rossi (1987). Numeri cromosomici per la flora Italiana: 1127-1135. Inform. Bot. Ital. 19: 314-318.

Castroviejo, S., M. Laínz, G. López González, P. Montserrat, F. Muñoz Garmendia, J. Paiva \& L. VIllar (eds.) (1986). Flora iberica. Plantas vasculares de la Península Ibérica e Islas Baleares, I. Real Jardín Botánico, CSIC. Madrid.

Clapham, A.R., T.G. Tutin \& D.M. Moore (1987). Flora of the British Isles, ed. 3. Cambridge.

COHEN, A.L. (1974). Critical point drying. In: M.A. Hayat (ed.), Principles and techniques of scanning electron microscopy l: 44-112. New York, Van Nostrand Reinhold.

CuÉnOD, A. (1954). Flore analytique et synoptique de la Tunisie. Tunis.

DAvis, P.H. (1984). Flora of Turkey and East Aegean Islands, vol. 8. Edinburgh.

DRESSLER, R.L. (1981). The Orchids. natural history and classification. Harvard Univ. Press. Cambridge, etc.

DruSKoviC, B. \& M. LovkA (1995). IOPB chromosome data 9. Int. Organ. Pl. Biosyst. Newslett. 24: 15-19.

FreudensteIN, J.V. \& F.N. RASMUSSEN (1999). What does morphology tell us about orchid relationships? - A cladistic analysis. Amer. J. Bot. 86: 225 248.

LOPEZ VéLEZ, G. (1984). Aportación al catálogo florístico de la Sierra del Calar del Mundo y sierras adyacen- 
tes del sur de Albacete (España) (1), Collect. Bot. (Barcelona) 15: 267-288.

LOSA, M \& P. MONTSERRAT (1950). Aportación al conocimiento de la flora de Andorra. Zaragoza.

LUER, C.A. (1975). The Native Orchids of the United States and Canada excluding Florida. The New Botanical Garden. New York.

MAIRE, R. (1959). Flore de l'Afrique du Nord, vol. 6. P. Lechevalier, Paris.

MLLER. P. (1768). The gardeners dictionary ed, 8. London.

MiEIETA, K. (1986). Karyological study of the Slovak flora XI. Acta Fac. Rerum Nat. Univ. Comenianae, Bot. 33: $51-55$.

Molero Mesa, J., F.A. Pérez Raya \& J.M. Martínez ParRas (1981). Relación de las Orquidaceae de la provincia de Granada. Anales Jard. Bot. Madrid 37(2): 645-659.

MoORE, D.M. (1980). Cephalanthera. In: T.G. Tutin, V.H. Heywood, N.A. Burgess, D.M. Moore, D.H. Valentine, S.M. Walters, D.A. Webb (eds.), Flora europaea 5: 328-329. Cambridge.

NAKAI, T. (1911). Flora koreana. Pars secunda. J. Coll. Sci. Imp. Univ. Tokyo 31: 1-573, 20 tab.

NAVARRo ANDRÉs, S. \& T.E. Díaz GonZÁlez (1977). Algunas novedades y anotaciones para la flora asturleonesa. Trab. Dept. Bot. Univ. Oviedo 1: 41-53.

NevsKII, S.A. (1935). Cephalanthera. In: V.L. Komarov (ed.), Flora of the USSR. Liliiflorae and Microspermae 4: 630-635. Botanicheskii Institut Akademii Nauk SSSR. Jerusalem [traducción inglesa].

ORIA DE RuEDA, J.A., J. DízZ \& M. RodríguEZ (1996). Guia de las plantas silvestres de Palencia. Ediciones Cálamo. Palencia.

Pallarés Navarro, A. (1999). Orquídeas de Almería. Almería.

Pérez Chiscano, J.L. (1977). Aportación al estudio de orquídeas de Extremadura. Anales Jard. Bot. Madrid 34(1): 175-181.

Prutsch, J. \& R. SCHILL (2001). The stigma of Cephalanthera (Orchidaceae) provides a link between primitive and derived Epidendroideae. Nordic J. Bot. 20(5): 599-604.

RENZ, J. (1978). Cephalanthera. In: K.H. Rechinger (ed.), Flora des iranischen hochlandes und der umrahmenden gebirge 126: 22-31. Akademische Druck-u. Verlagsanstalt Graz-Austria.

RuIz, L. (1995). Mediterranean chromosome number reports 5 (559-589). Fl. Medit. 5: 363-373.

SemERENKo, L.V. (1989). Chromosome numbers in some members of the families Asteraceae, Fabaceae, Orchidaceae and Poaceae from the Berezinsky Biosphere. Bot. Zhurn. (Moscow \& Leningrad) 74: 1671-1673.

SuÁREZ, R. \& M.J. DIEZ (1987). Orchidaceae. In: B. Valdés, M.J. Díez \& I. Fernández (eds.), Atlas polínico de Andalucia Occidental: 407. Cádiz.

VALLE, F. (1981). Contribución al estudio fitosociológico de las Sierras de Alfacar y Huétor (Granada-España). Anales Jard. Bot. Madrid 37(2): 725-736.

VERLAQUE, R.. M. SEIDENBINDER \& C. RaYNAUd (1987). Recherches cytotaxonomiques sur la spéciation en région Méditerranéenne III: espèces aneuploides. Biol. \& Écol. Médit. 10: 315-346.
VIJ, S.P. \& R. SoOD (1986). Cytology in relation to morphology in Spiranthes lancea (Sw.) Backer. In: S.P. Vij (ed.), Biology, Conservation, and Culture of Orchids: 147-161. New Delhi.

WillKomm, M. (1861). Cephalanthera. In: M. Willkomm \& J. Lange (eds.), Prodromus Florae Hispanicae 1: 175-176. Stuttgartiae.

Zhu Guanghua (1999). Cephalanthera. In: Lang Kaiyong (ed.), Flora Reipublicae Populari Sinicae 17: 74-81. Institutum Botanicum Academiae Sinicae. Science Press.

ZokU, K.R. (1965). Cephalanthera L.C. Rich. In: J. Ohwi (ed.), Flora of Japan. Washington, D.C. [edición inglesa].

\section{ÍNDICE DE NOMBRES CIENTÍFICOS}

Cephalanthera Rich., 227, 228, 231

alba (Crantz) Simonk., 241

alpicola Fukuy., 227

angustifolia Simonk., 236

austinae (Gray) Heller, 227

bijianjensis S.C. Chen, 227

calcarata S.C. Chen \& K.Y. Lang, 227

caucasica Kraenzl., 227

cesari-gonzalezii Rivas Goday \& Bellot, 236

cucullata Boiss \& Heldr., 228

damasonium (Mill.) Druce. 228, 229, 230,

231,241

ensifolia Rich., 236

var. cesari-gonzalezii Rivas Goday \& Bellot, 236

epipactoides Fisch. \& C.A. Mey., 227

erecta (Thunb. ex Murray) Blume, 227

falcata (Thunb. ex Murray) Blume, 227

grandiflora auct., 241

grandiflora Gray, 236

kotschyana Renz \& Taubenheim, 227

kurdica Borm. \& Kraenzl., 227

lancifolia (Murray) Tod., 241

latifolia (Mill.) Janch., 241

longibracteata Blume, 227

longifolia (L.) Fritsch, 228, 229, 230, 231, 236

f. angustifolia Maire \& Weiller, 236

f. latifolia (Maire) Maire, 236

var. latifolia (Maire) D. Rivera \& López

Vélez, 236

var. pilosa Harz in Schltdl., 236

ochroleuca (Baumg.) Rchb., 241

pallens (Sw.) Rich., 236

rubra (L.) Rich., 228, 229, 230, 231

segobricensis Pau, 236

xiphophyllum (Ehrh. ex L. f.) Rchb. f., 236

var. latifolia Maire, 236

Epipactis Sw. alba Crantz, 241 
lancifolia (Murray) All., 241

grandiflora L., 236

longifolia (L.) Wettst., 236

ochroleuca Baumg., 241

pallens Sw., 236

purpurea Crantz, 231

rubra (L.) All., 231

Serapias L.

damasonium Mill., 241

ensifolia Murray, 236

grandiflora L., 236

helleborine

var. longifolia L., 236 lancifolia Murray, 241

latifolia Mill., 241

lonchophyllum L. f., 236

longifolia (L.) Huds., 236

nivea Desf., 236

nivea Vill., 236

pallens Jundz., 241

pallida Wahlenb., 236

rubra L., 231

xiphophyllum Ehrh. ex L. f., 236

Editado por Roberto Gamarra Aceptado para publicación: 30-I-2002 